\title{
Weighted Gaussian entropy and determinant inequalities
}

\author{
Y. Suhov ${ }^{1-3}$, S. Yasaei Sekeh ${ }^{4}$ I. Stuhl ${ }^{5-7}$ \\ ${ }^{1}$ DPMMS, University of Cambridge, UK \\ 2 Math Dept, Penn State University, PA, USA \\ ${ }^{3}$ IPIT RAS, Moscow, RF \\ ${ }^{4}$ DEs, Federal University of São Carlos, SP, Brazil \\ ${ }^{5}$ IMS, University of São Paulo, SP, Brazil \\ ${ }^{6}$ Math Dept, University of Denver, CO, USA \\ ${ }^{7}$ University of Debrecen, Hungary
}

\begin{abstract}
We produce a series of results extending information-theoretical inequalities (discussed by Dembo-Cover-Thomas in 1989-1991) to a weighted version of entropy. The resulting inequalities involve the Gaussian weighted entropy; they imply a number of new relations for determinants of positive-definite matrices.
\end{abstract}

\section{Introduction}

The aim of this paper is to give a number of new bounds involving determinants of positivedefinite matrices. These bounds can be considered as generalizations of inequalities discussed in [2, 5]. A common feature of determinant inequalities (DIs) from [2, 5] is that most of them have been previously known but often proven by individual arguments (see the bibliography in [2, 5]). The unifying approach adopted in [2, 5] emphasized their common nature connected with/through information-theoretical entropies.

The bounds presented in the current paper are also obtained by a unified method which is based on weighed entropies (WEs), more precisely, on Gaussian WEs. Hence, we speak here of weighted determinant bounds/inequalities. The weighted determinant inequalities (WDIs) offered in the present paper are novel, at least to the best of our knowledge. Moreover, when we choose the weight function to be a (positive) constant, a WDI become a 'standard' DI. In fact, the essence of this work is that we subsequently examined DIs from [2, 5, for a possibility of a (direct) extension to non-constant weight functions; successful attempts formed the present paper. This reflects a particular feature of the present paper: a host of new inequalities are obtained by an old method while [2, 5] re-establish old inequalities by using a new method.

As a primary example, consider the so-called Ky Fan inequality. (We follow the terminology used in [2, 5, 3].) This inequality asserts that $\delta(\mathbf{C}):=\log \operatorname{det} \mathbf{C}$ is a concave function of a

2010 Mathematics Subject Classification: 60A10, 60B05, 60C05.

Key words and phrases: weight function, weighted entropy, weighted conditional and mutual entropies, weighted Gaussian entropy, weighted determinant inequalities 
positive-definite $d \times d$ matrix $\mathbf{C}$. In other words, for all strictly positive-definite $d \times d$ matrices $\mathbf{C}_{1}, \mathbf{C}_{2}$ and $\lambda_{1}, \lambda_{2} \geq 0$ with $\lambda_{1}+\lambda_{2}=1$

$$
\delta\left(\lambda_{1} \mathbf{C}_{1}+\lambda_{2} \mathbf{C}_{2}\right)-\lambda_{1} \delta\left(\mathbf{C}_{1}\right)-\lambda_{2} \delta\left(\mathbf{C}_{2}\right) \geq 0 ; \text { equality iff } \lambda_{1} \lambda_{2}=0 .
$$

For original 'geometric' proofs of (1.1) and other related inequalities, see Ref [8] and the bibliography therein. In [2, 5, 3] the derivation of (1.1) occupies few lines and is based on the fact that under a variance constraint, the differential entropy is maximized at a Gaussian density.

A weighted Ky Fan inequality (1.2) has been proposed in 9], Theorem 3.2; the derivation is also short and based on a maximization property of the weighted entropy (cf. Theorem 3.1 below). Namely, given $\mathbf{C}_{1}, \mathbf{C}_{2}$ and $\lambda_{1}, \lambda_{2}$ as above and a nonnegative function $\mathbf{x} \in \mathbb{R}^{d} \mapsto \phi(\mathbf{x})$, positive on an open domain in $\mathbb{R}^{d}$, assume condition (1.6). Then

$$
\sigma\left(\lambda_{1} \mathbf{C}_{1}+\lambda_{2} \mathbf{C}_{2}\right)-\lambda_{1} \sigma\left(\mathbf{C}_{1}\right)-\lambda_{2} \sigma\left(\mathbf{C}_{2}\right) \geq 0 ; \text { equality again iff } \lambda_{1} \lambda_{2}=0 .
$$

Here, for a strictly positive-definite $\mathbf{C}$, the value $\sigma(\mathbf{C})=\sigma_{\phi}(\mathbf{C})$ is as follows:

$$
\sigma_{\phi}(\mathbf{C})=\frac{\alpha_{\phi}(\mathbf{C})}{2} \log \left[(2 \pi)^{d}(\operatorname{det} \mathbf{C})\right]+\frac{\log e}{2} \operatorname{tr} \mathbf{C}^{-1} \mathbf{\Phi}_{\mathbf{C}, \phi}:=h_{\phi}^{\mathrm{w}}\left(f_{\mathbf{C}}^{\mathrm{No}}\right)
$$

Next, $\alpha_{\phi}(\mathbf{C})>0$ and positive-definite matrix $\mathbf{\Phi}_{\mathbf{C}, \phi}$ are given by

$$
\alpha_{\phi}(\mathbf{C})=\int_{\mathbb{R}^{d}} \phi\left(\mathbf{x}_{1}^{d}\right) f_{\mathbf{C}}^{\mathrm{No}}\left(\mathbf{x}_{1}^{d}\right) \mathrm{d} \mathbf{x}_{1}^{d}, \quad \mathbf{\Phi}_{\mathbf{C}, \phi}=\int_{\mathbb{R}^{d}} \mathbf{x}_{1}^{d}\left(\mathbf{x}_{1}^{d}\right)^{\mathrm{T}} \phi\left(\mathbf{x}_{1}^{d}\right) f_{\mathbf{C}}^{\mathrm{No}}\left(\mathbf{x}_{1}^{d}\right) \mathrm{d} \mathbf{x}_{1}^{d},
$$

and $f_{\mathbf{C}}^{\mathrm{No}}$ stands for a normal probability density function $(\mathrm{PDF})$ with mean $\mathbf{0}$ and covariance matrix $\mathbf{C}$ :

$$
f_{\mathbf{C}}^{\mathrm{No}}(\mathbf{x})=\frac{1}{(2 \pi)^{d / 2}(\operatorname{det} \mathbf{C})^{1 / 2}} \exp \left(-\frac{1}{2} \mathbf{x}^{\mathrm{T}} \mathbf{C}^{-1} \mathbf{x}\right), \quad \mathbf{x}=\left(\begin{array}{c}
x_{1} \\
\vdots \\
x_{n}
\end{array}\right) \in \mathbb{R}^{d} .
$$

In terms of a multivariate normal random vector $\mathbf{X}_{1}^{d} \sim f_{\mathbf{C}}^{\text {No }:} \alpha_{\phi}(\mathbf{C})=\mathbb{E} \phi\left(\mathbf{X}_{1}^{d}\right)$ and $\boldsymbol{\Phi}_{\phi, \mathbf{C}}=$ $\mathbb{E}\left(\phi\left(\mathbf{X}_{1}^{d}\right)\left[\mathbf{X}_{1}^{d}\left(\mathbf{X}_{1}^{d}\right)^{\mathrm{T}}\right]\right)$. In (1.5) and below we routinely omit the indices in the notation like $\mathbf{x}_{1}^{d}$ and $\mathbf{X}_{1}^{d}$. The quantity $h_{\phi}^{\mathrm{w}}\left(f_{\mathbf{C}}^{\mathrm{No}}\right)=-\int_{\mathbb{R}^{d}} \phi(\mathbf{x}) f_{\mathbf{C}}^{\mathrm{No}}(\mathbf{x}) \log f_{\mathbf{C}}^{\mathrm{No}}(\mathbf{x}) \mathrm{d} \mathbf{x}$ is the weighted entropy of $f_{\mathbf{C}}^{\mathrm{No}}$ with weight function $\phi$, a concept analyzed in detail below. For $\phi(\mathbf{x}) \equiv 1, h_{\phi}^{\mathrm{w}}\left(f_{\mathbf{C}}^{\mathrm{No}}\right)$ coincides with a 'standard' (differential) entropy of a normal PDF.

The assumption upon $\mathbf{C}_{1}, \mathbf{C}_{2}$ and $\lambda_{1}, \lambda_{2}$ consists of two bounds and reads

$$
\begin{gathered}
\lambda_{1} \alpha\left(\mathbf{C}_{1}\right)+\lambda_{2} \alpha\left(\mathbf{C}_{2}\right)-\alpha\left(\lambda_{1} \mathbf{C}_{1}+\lambda_{2} \mathbf{C}_{2}\right) \geq 0 \\
{\left[\lambda_{1} \alpha\left(\mathbf{C}_{1}\right)+\lambda_{2} \alpha\left(\mathbf{C}_{2}\right)-\alpha\left(\lambda_{1} \mathbf{C}_{1}+\lambda_{2} \mathbf{C}_{2}\right)\right]} \\
\times \log \left\{(2 \pi)^{d}\left[\operatorname{det}\left(\lambda_{1} \mathbf{C}_{1}+\lambda_{2} \mathbf{C}_{2}\right)\right]\right\}+\operatorname{tr}\left[\left(\lambda_{1} \mathbf{C}_{1}+\lambda_{2} \mathbf{C}_{2}\right)^{-1} \boldsymbol{\Delta}\right] \leq 0
\end{gathered}
$$

where matrix $\boldsymbol{\Delta}=\lambda_{1} \boldsymbol{\Phi}_{\mathbf{C}_{1}}+\lambda_{2} \boldsymbol{\Phi}_{\mathbf{C}_{2}}-\boldsymbol{\Phi}_{\lambda_{1} \mathbf{C}_{1}+\lambda_{2} \mathbf{C}_{2}}$. Bounds (1.6) have opposite directions and stem from the weighted Gibbs inequality. Cf. Eqns (1.3), (3.3) from Ref [9] and (3.1), (3.5) from Section 3 below. 
When $\phi(\mathbf{x}) \equiv 1$, Eqn (1.6) is satisfied: we have equalities. In this case the weighted Ky Fan inequality (1.2) transforms into (1.1). In general, condition (1.6) is not trivial: in a simplified case of an exponential weight function $\phi(\mathbf{x})=\exp \left(\mathbf{t}^{\mathrm{T}} \mathbf{x}\right), \mathbf{t} \in \mathbb{R}^{d}$, it has been analyzed, both analytically and numerically, in [10]. (Here, $\phi(\mathbf{x}) \equiv 1$ means $\mathbf{t}=\mathbf{0}$.) As was shown in [10], for given $\mathbf{C}_{1}, \mathbf{C}_{2}, \lambda_{1}, \lambda_{2}$ and $\phi$ (that is, for a given $\mathbf{t}$ ), Eqn (1.6) may or may not be fulfilled. (And when (1.6) fails, (1.2) may still hold true.) Moreover, when (1.6) holds, it may or may not produce a strictly positive expression in the RHS of bound (1.1). (Thus, in some cases we can speak of an improvement in the Ky Fan inequality.) See Ref [10]. We believe that further studies in this direction should follow, focusing on specific forms of weight function $\phi$.

In our opinion, this paper paves way to a similar analysis of the whole host of newly established WDIs. These inequalities should be taken with a justified degree of caution: offered sufficient conditions (stated in the form of bounds involving various weight function) may fail for particular $\mathbf{C}_{1}, \mathbf{C}_{2}, \lambda_{1}, \lambda_{2}$, and $\phi$, and a given WDI may or may not yield an improvement compared to its 'standard' counterpart. For reader's convenience we list the sufficient conditions figuring across the paper: Eqns (2.8), (2.20), (3.1), (3.5), (4.1), (4.4) (4.7), (4.10), (5.3), (5.12), (5.20), (5.24), (6.3), (6.11), (6.12) and (6.17).

The presented WDIs generalize what is sometimes called elementary information-theoretic inequalities. An opposite example is the entropy-power inequality; and related bounds. Here the intuition is more intricate; some initial results have been proposed in [11].

The paper is organized as follows. In Section 2 we work with a general setting, elaborating on properties of weighted entropies which have been established earlier in [9]. Section 3 summarizes some properties of Gaussian weighted entropies while Section 4 analyzes the behavior of weighted entropies under mappings; these sections also rely on Ref. [9. The WDIs are presented in Sections 5 and 6 as a sequel to the material from Section 2 - 4. Again, for reader's convenience we list them here as Eqns (5.4), (5.13), (5.14), (5.21), (5.25), (5.26), (6.5), (6.14) and (6.18).

\section{Random strings and reduced weight functions}

The WE of a probability distribution was introduced in late 1960s - early 1970s; see, e.g., [1]. (Another term that can be used is a context-dependent or a preferential entropy.) The reader is referred to [9] where a number of notions and elementary inequalities were established for the WE, mirroring well-known facts about the standard (Shannon) entropy. We also use Refs [2, 5] as a source of standard inequalities which we extend to the case of the WE. To keep pre-emptiveness, we follow the system of notation from [2, 5, 9] with minor deviations.

Let us begin with general definitions. The WE of a random element $X$ taking values in a standard measure space (SMS) $(\mathcal{X}, \mathfrak{M}, \nu)$ with a weight function (WF) $x \in \mathcal{X} \mapsto \phi(x) \geq 0$ is defined by

$$
h_{\phi}^{\mathrm{w}}(X)=h_{\phi}^{\mathrm{w}}(f)=\mathbb{E}(\phi(X) \log f(X))=-\int_{\mathcal{X}} \phi(x) f(x) \log f(x) \nu(\mathrm{d} x),
$$

assuming that $\phi$ is measurable and the integral is absolutely convergent. Here $f=f_{X}$ is the probability mass/density function $(\mathrm{PM} / \mathrm{DF})$ of $X$ relative to measure $\nu$. Symbol $\mathbb{E}$ stands for the expected value (relative to a probability distribution that is explicitly specified or emerges from the context in an unambiguous manner). 
A number of properties of the WE are related to a Cartesian product structure. Let random elements $X_{1}, \ldots, X_{n}$ be given, taking values in $\operatorname{SMSs}\left(\mathcal{X}_{i}, \mathfrak{M}_{i}, \nu_{i}\right), 1 \leq i \leq n$. Set $\underline{X}_{1}^{n}:=$ $\left\{X_{1}, \ldots, X_{n}\right\}$ and assume that $X_{1}, \ldots, X_{n}$ have a joint PM/DF $f_{\underline{X}_{1}^{n}}\left(\underline{x}_{1}^{n}\right), \underline{x}_{1}^{n} \in \mathcal{X}_{1}^{n}:=\underset{1 \leq i \leq n}{\overline{\times}} \mathcal{X}_{i}$, relative to the measure $\nu_{1}^{n}:=\underset{1 \leq i \leq n}{\times} \nu_{i}$; for brevity we will sometimes set $f_{\underline{X}_{1}^{n}}=f$. The joint WE of string $\underline{X}_{1}^{n}$ is defined as

$$
h_{\phi}^{\mathrm{W}}\left(\underline{X}_{1}^{n}\right)=-\mathbb{E}\left(\phi\left(\underline{X}_{1}^{n}\right) \log f\left(\underline{X}_{1}^{n}\right)\right)=-\int_{\mathcal{X}_{1}^{n}} \phi\left(\underline{x}_{1}^{n}\right) f\left(\underline{x}_{1}^{n}\right) \log f\left(\underline{x}_{1}^{n}\right) \nu_{1}^{n}\left(\mathrm{~d} \underline{x}_{1}^{n}\right) .
$$

Given a set $S \subseteq I:=\{1,2, \ldots, n\}$, write

$$
\underline{X}(S), \underline{X}\left(S^{\complement}\right) \text { for strings }\left\{X_{i}: i \in S\right\},\left\{X_{i}: i \in S^{\complement}\right\} \text {, respectively, where } S^{\complement}=I \backslash S \text {. }
$$

Next, let $\underline{x}(S)$ and $\underline{x}\left(S^{\complement}\right)$ stand for

$$
\left\{x_{i}: i \in S\right\} \in \mathcal{X}(S):=\underset{i \in S}{\times} \mathcal{X}_{i} \text { and }\left\{x_{i}: i \in S^{\complement}\right\} \in \mathcal{X}\left(S^{\complement}\right):=\underset{i \in S^{\complement}}{\times} \mathcal{X}_{i} .
$$

Accordingly, the marginal $\mathrm{PD} / \mathrm{MF} f_{\underline{X}(S)}(\underline{x}(S))$ emerges, for which we will often write $f_{S}(\underline{x}(S))$ or even $f(\underline{x}(S))$ for short. Furthermore, given a WF $\underline{x}_{1}^{n} \mapsto \phi\left(\underline{x}_{1}^{n}\right) \geq 0$, we define the function $\psi(S): \underline{x}(S) \mapsto \psi(S ; \underline{x}(S)) \geq 0$ involving the conditional PM/DF $f_{\underline{X}\left(S^{\complement}\right) \mid \underline{X}(S)}\left(\underline{x}\left(S^{\complement}\right) \mid \underline{x}(S)\right):$

$$
\psi(S ; \underline{x}(S))=\int_{\mathcal{X}\left(S^{\complement}\right)} \phi\left(\underline{x}_{1}^{n}\right) \underline{f}_{\underline{X}\left(S^{\complement}\right) \mid \underline{X}(S)}\left(\underline{x}\left(S^{\complement}\right) \mid \underline{x}(S)\right) \nu_{\mathcal{X}\left(S^{\complement}\right)}\left(\mathrm{d} \underline{x}\left(S^{\complement}\right)\right)
$$

where $\nu_{\mathcal{X}\left(S^{\complement}\right)}:=\underset{i \in S^{\complement}}{\times} \nu_{i}$. For brevity we again write sometimes $f_{S^{\complement} \mid S}$ instead of $f_{\underline{X}\left(S^{\complement}\right) \mid \underline{X}(S)}$ or omit subscripts altogether. We also write $\mathrm{d} \underline{x}(S)$ and $\mathrm{d} \underline{x}\left(S^{\complement}\right)$ instead of $\nu_{\mathcal{X}(S)}(\mathrm{d} \underline{x}(S))$ and $\nu_{\mathcal{X}(S)}\left(\mathrm{d} \underline{x}\left(S^{\complement}\right)\right)$ and $\mathrm{d} \underline{x}$ instead of $\nu_{1}^{n}\left(\mathrm{~d} \underline{x}_{1}^{n}\right)$.

Function $\psi(S ; \cdot)$ will play the role of a reduced (or induced) WF when we pass from $\underline{X}_{1}^{n}$ to a sub-string $\underline{X}(S)$. More precisely, set

$$
\begin{aligned}
h_{\psi(S)}^{\mathrm{w}}(\underline{X}(S)) & =-\mathbb{E}\left(\psi\left(S ; \underline{X}(S) \log f_{S}(S ; \underline{X}(S))\right)\right. \\
& =-\int_{\mathcal{X}(S)} \psi(S ; \underline{x}(S)) f_{S}(\underline{x}(S)) \log f_{S}(\underline{x}(S)) \mathrm{d} \underline{x}(S),
\end{aligned}
$$

with $\nu_{\mathcal{X}(S)}:=\underset{i \in S}{\times} \nu_{i}$. Cf. [9]. Next, for $k=1, \ldots, n$ define

$$
h_{k}^{\mathrm{w}, n}=\left(\begin{array}{l}
n \\
k
\end{array}\right)^{-1} \sum_{S \subseteq I: \#(S)=k} \frac{h_{\psi(S)}^{\mathrm{w}}(\underline{X}(S))}{k} .
$$

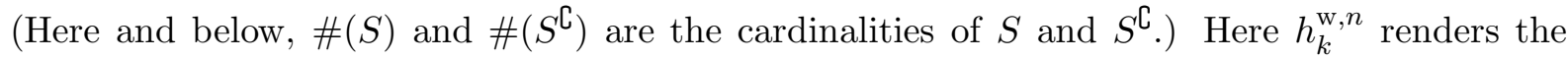
averaged WE (per string and per element) of a randomly drawn $k$-element sub-string in $\underline{X}_{1}^{n}$. 
In what follows we use the concepts of the conditional and mutual WE and their properties; cf. [9]. These objects are used with a host of WFs, depending on the context. Consider the following condition:

$$
\begin{aligned}
& \forall i \in S \subseteq I, \text { with } S_{i}^{-}=\{j \in S: j<i\} \text { and } S_{i}^{+}=\{j \in S: j>i\} \\
& \quad \int_{\mathcal{X}(S)} \psi(S ; \underline{x}(S))\left\{f(\underline{x}(S))-f\left(\underline{x}\left(S_{i}^{-}\right)\right) \times\left[f\left(x_{i} \mid \underline{x}\left(S_{i}^{-}\right)\right) f\left(\underline{x}\left(S_{i}^{+}\right) \mid \underline{x}\left(S_{i}^{-}\right)\right)\right]\right\} \mathrm{d} \underline{x}(S) \geq 0,
\end{aligned}
$$

with standard agreements when one of the sets $S_{i}^{ \pm}=\emptyset$. Pictorially, Eqn (2.8) is an extension of bound (1.27) from [9]; it means that for all $i \in S \subseteq I$, the induced WF $\psi(S ; \cdot)$ is correlated more positively with the marginal PM/DF $f_{S}(\underline{x}(S))$ than with the dependence-broken product $f_{S_{i}^{-}}\left(\underline{x}\left(S_{i}^{-}\right)\right) \times\left[f_{\{i\} \mid S_{i}^{-}}\left(x_{i} \mid \underline{x}\left(S_{i}^{-}\right)\right) f_{S_{i}^{+} \mid S_{i}^{-}}\left(\underline{x}\left(S_{i}^{+}\right) \mid \underline{x}\left(S_{i}^{-}\right)\right)\right]$. Another version of (essentially) the same property is Eqn (2.20) below.

Remark 2.1 The special choice of sets $S_{i}^{ \pm}$is not particularly important: it can be a general partition of $S \backslash\{i\}$ allowing us to use the chain rule for the conditional WE (see below).

Theorem 2.2 (Cf. 2], Lemma 7 or [5], Theorem 1.) Let $h_{k}^{\mathrm{w}, n}$ be defined as in (2.7) and assume (2.8). Then

$$
h_{1}^{\mathrm{w}, n} \geq h_{2}^{\mathrm{w}, n} \geq \ldots \geq h_{n-1}^{\mathrm{w}, n} \geq h_{n}^{\mathrm{w}, n} .
$$

Proof. Begin with the last inequality, $h_{n-1}^{\mathrm{w}, n} \geq h_{n}^{\mathrm{w}, n}$. Let $1 \leq i \leq n$ and choose $S=I$, $S_{i}^{-}=I_{i}^{-}:=\{1, \ldots, i-1\}$ and $S_{i}^{+}=I_{i}^{+}:=\{i+1, \ldots, n\}$, with $\{i\}^{\complement}=I_{i}^{-} \cup I_{i}^{+}($cf. (2.3) $)$(2.4 $)$. Then the condition

$$
\left.\int_{\left(\mathcal{X}^{n}\right)^{1}} \phi(\underline{x})\left[f(\underline{x})-f_{\underline{X}_{1}^{i-1}}\left(\underline{x}_{1}^{i-1}\right) f\left(x_{i} \mid \underline{x}_{1}^{i-1}\right) f\left(\underline{x}_{i+1}^{n} \mid \underline{x}_{1}^{i-1}\right)\right] \mathrm{d} \underline{x} \geq 0 \quad \text { (by virtue of (2.8) }\right) \text {, }
$$

yields:

$$
\begin{aligned}
h_{\phi}^{\mathrm{w}}\left(\underline{X}_{1}^{n}\right) & =h_{\phi}^{\mathrm{w}}\left(X_{i} \mid \underline{X}\left(\{i\}^{\complement}\right)+h_{\psi\left(\{i\}^{\complement}\right)}^{\mathrm{w}}\left(\underline{X}\left(\{i\}^{\complement}\right)\right) \quad\right. \text { by the chain rule } \\
& \leq h_{\psi\left(I_{i}^{-}\right)}^{\mathrm{w}}\left(X_{i} \mid \underline{X}_{1}^{i-1}\right)+h_{\psi\left(\{i\}^{\complement}\right)}^{\mathrm{w}}\left(\underline{X}\left(\{i\}^{\complement}\right)\right) \quad \text { by Lemma } 1.3 \text { from [9]. }
\end{aligned}
$$

Here reduced WFs $\psi\left(\{i\}^{\complement}\right)$ and $\psi\left(I_{i}^{-}\right)$are calculated according to the recipies in (2.5), (2.6).

Taking the sum, we obtain:

$$
n h_{\phi}^{\mathrm{w}}\left(\underline{X}_{1}^{n}\right) \leq \sum_{i=1}^{n} h_{\psi\left(\{i\}^{\complement}\right)}^{\mathrm{w}}\left(\underline{X}\left(\{i\}^{\complement}\right)+\sum_{i=1}^{n} h_{\psi\left(I_{i}^{-}\right)}^{\mathrm{w}}\left(X_{i} \mid \underline{X}_{1}^{i-1}\right) .\right.
$$

By using the chain rule, $\sum_{i=1}^{n} h_{\psi\left(I_{i}^{-}\right)}^{\mathrm{w}}\left(X_{i} \mid \underline{X}_{1}^{i-1}\right)=h_{\phi}^{\mathrm{w}}\left(\underline{X}_{1}^{n}\right)$. Hence, Eqn (2.10) becomes

$$
(n-1) h_{\phi}^{\mathrm{w}}\left(\underline{X}_{1}^{n}\right) \leq \sum_{i=1}^{n} h_{\psi\left(\{i\}^{\complement}\right)}^{\mathrm{w}}\left(\underline{X}\left(\{i\}^{\complement}\right) .\right.
$$


Consequently,

$$
h_{\phi}^{\mathrm{w}}\left(\underline{X}_{1}^{n}\right) \leq \sum_{i=1}^{n} \frac{h_{\psi\left(\{i\}^{\complement}\right)}^{\mathrm{w}}\left(\underline{X}\left(\{i\}^{\complement}\right)\right.}{n-1},
$$

which yields that $h_{n-1}^{\mathrm{w}, n-1} \geq h_{n}^{\mathrm{w}, n}$.

This argument can be repeated if we restrict the WE and the PM/DF to a $k$-element subset $S=\left\{i_{1}, \ldots, i_{k}\right\} \subset I$ listed in an increasing order of its points and perform a uniform choice over its $(k-1)$-elements subsets. Condition (2.8) yields the bound

$$
\frac{1}{k} h_{\psi(S)}^{\mathrm{w}}(\underline{X}(S)) \leq \frac{1}{k} \sum_{i \in S} \frac{h_{\psi(S \backslash\{i\})}^{\mathrm{w}}(\underline{X}(S \backslash\{i\}))}{k-1} .
$$

Hence for each $k$-element subset, $h_{k-1}^{\mathrm{w}, k} \geq h_{k}^{\mathrm{w}, k}$. Therefore, the inequality remains true after taking the average over all $k$-element subsets drawn uniformly.

In Theorem 2.3 we extend the result of Theorem 2.2 to exponents of WEs for sub-strings in $\underline{X}_{1}^{n}$.

Theorem 2.3 (Cf. [2], Corollary of Lemma 7 or [5], Corollary 1) Given $r>0$, define:

$$
g_{k}^{\mathrm{w}, n}=\left(\begin{array}{l}
n \\
k
\end{array}\right)^{-1} \sum_{S \subseteq I: \#(S)=k} \exp \left[r \frac{h_{\psi(S)}^{\mathrm{w}}(\underline{X}(S))}{k}\right] .
$$

Then, under assumption (2.8),

$$
g_{1}^{\mathrm{w}, n} \geq g_{2}^{\mathrm{w}, n} \geq \cdots \geq g_{n-1}^{\mathrm{w}, n} \geq g_{n}^{\mathrm{w}, n} .
$$

Proof. Again, it is convenient to start with the last bound in (2.13). As in [2], multiply Eqn (2.11) by $r$, exponentiate and apply the arithmetic-geometric mean inequality to obtain $g_{n-1}^{\mathrm{w}, n} \geq g_{n}^{\mathrm{w}, n}$. The result is then completed with the help of same argument as in the proof of Theorem 2.2

In Theorem 2.4 we analyse the averaged conditional WEs for sub-strings in $\underline{X}_{1}^{n}$.

Theorem 2.4 (Cf. [5], Theorem 2.) Let $p_{k}^{\mathrm{w}, n}$ be defined as

$$
p_{k}^{\mathrm{w}, n}=\left(\begin{array}{l}
n \\
k
\end{array}\right)^{-1} \sum_{S \subseteq I: \#(S)=k} \frac{h_{\phi}^{\mathrm{w}}\left(\underline{X}(S) \mid \underline{X}\left(S^{\complement}\right)\right)}{k} .
$$

Then under the assumption

$$
\int_{\mathcal{X}_{1}^{n}} \phi(\underline{x})\left[f(\underline{x})-\prod_{i=1}^{n} f\left(x_{i}\right)\right] \mathrm{d} \underline{x} \geq 0
$$

we have that

$$
p_{1}^{\mathrm{w}, n} \leq p_{2}^{\mathrm{w}, n} \leq \ldots \leq p_{n-1}^{\mathrm{w}, n} \leq p_{n}^{\mathrm{w}, n}
$$


Proof. Following the argument used in [9], Theorem 3.1, condition (2.15) yields

$$
h_{\phi}^{\mathrm{w}}\left(\underline{X}_{1}^{n}\right) \leq \sum_{i=1}^{n} h_{\psi(\{i\})}^{\mathrm{w}}\left(X_{i}\right) .
$$

Subtracting both sides from $n h_{\phi}^{\mathrm{w}}\left(\underline{X}_{1}^{n}\right)$, we obtain:

$$
(n-1) h_{\phi}^{\mathrm{w}}\left(\underline{X}_{1}^{n}\right) \geq \sum_{i=1}^{n}\left[h_{\phi}^{\mathrm{w}}\left(\underline{X}_{1}^{n}\right)-h_{\psi(\{i\})}^{\mathrm{w}}\left(X_{i}\right)\right],
$$

By the conditional WE definition,

$$
h_{\phi}^{\mathrm{w}}\left(\underline{X}_{1}^{n}\right)=h_{\phi}^{\mathrm{w}}\left(\underline{X}_{1}^{i-1}, \underline{X}_{i+1}^{n} \mid X_{i}\right)+h_{\psi(\{i\})}^{\mathrm{w}}\left(X_{i}\right) .
$$

Hence,

$$
(n-1) h_{\phi}^{\mathrm{w}}\left(\underline{X}_{1}^{n}\right) \geq \sum_{i=1}^{n} h_{\phi}^{\mathrm{w}}\left(\underline{X}_{1}^{i-1}, \underline{X}_{i+1}^{n} \mid X_{i}\right)
$$

Dividing (2.17) by $n(n-1)$ yields that $p_{n-1}^{\mathrm{w}, n} \leq p_{n}^{\mathrm{w}, n}$. Finally, applying the same argument as in Theorem 2.2 completes the proof.

The next step is to pass to mutual WEs.

Theorem 2.5 (Cf. [5], Corollary 2.) Consider the averaged mutual WE between a subset (or a sub-string) and its complement:

$$
q_{k}^{\mathrm{w}, n}=\left(\begin{array}{l}
n \\
k
\end{array}\right)^{-1} \sum_{S \subseteq I: \#(S)=k} \frac{i_{\phi}^{\mathrm{w}}\left(\underline{X}(S): \underline{X}\left(S^{\complement}\right)\right)}{k},
$$

and assume (2.8). Then

$$
q_{1}^{\mathrm{w}, n} \geq q_{2}^{\mathrm{w}, n} \geq \ldots \geq q_{n-1}^{\mathrm{w}, n} \geq q_{n}^{\mathrm{w}, n} .
$$

Proof. The result is straightforward, from Theorems 2.2 and 2.4 and the following relation between conditional and mutual WEs:

$$
i_{\phi}^{\mathrm{w}}\left(\underline{X}(S): \underline{X}\left(S^{\complement}\right)\right)=h_{\psi(S)}^{\mathrm{w}}(\underline{X}(S))-h_{\phi}^{\mathrm{w}}\left(\underline{X}(S) \mid \underline{X}\left(S^{\complement}\right)\right) .
$$

In Theorem 2.6 we consider the following condition: for all set $S$ with $\# S \geq 2$ and $i, j \in S$ with $i \neq j$,

$$
\begin{aligned}
\int_{\mathcal{X}_{1}^{n}} \phi(\underline{x}) f & \left.\underline{x}\left(S^{\complement}\right) \mid \underline{x}(S)\right)[f(\underline{x}(S)) \\
& \left.-f(\underline{x}(S \backslash\{i, j\})) f\left(x_{i} \mid \underline{x}(S \backslash\{i, j\})\right) f\left(x_{j} \mid \underline{x}(S \backslash\{i, j\})\right)\right] \mathrm{d} \underline{x} \geq 0 .
\end{aligned}
$$

The meaning of (2.20) is that for all $S$ and $i, j$ as above, the reduced WF $\psi_{S}(x(S))$ is correlated

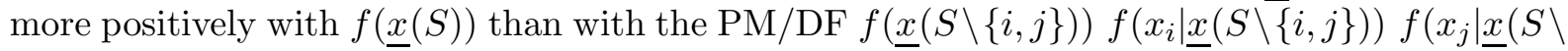
$\{i, j\})$ ) where the conditional dependence between $X_{i}$ and $X_{j}$ is broken, given $\underline{X}(S \backslash\{i, j\})$. 
Theorem 2.6 (Cf. [5], Theorem 3.) Define the average mutual WE as

$$
I_{k}^{\mathrm{w}, n}=\left(\begin{array}{l}
n \\
k
\end{array}\right)^{-1} \sum_{S \subseteq I: \#(S)=k} i_{\phi}^{\mathrm{w}}\left(\underline{X}(S): \underline{X}\left(S^{\complement}\right)\right) .
$$

By symmetry of the mutual WE, $I_{k}^{\mathrm{w}, n}=I_{n-k}^{\mathrm{w}, n}$. Assume condition (2.20). Then

$$
I_{1}^{\mathrm{w}, n} \leq I_{2}^{\mathrm{w}, n} \leq \ldots \leq I_{\lfloor n / 2\rfloor}^{\mathrm{w}, n} .
$$

Proof. Let $k \leq\lfloor n / 2\rfloor$. If $S$ is a subset of size $k$ then $S$ has $k$ subsets of size $k-1$. Thus, we write:

$$
\begin{aligned}
k i_{\phi}^{\mathrm{w}} & {\left[\underline{X}(S): \underline{X}\left(S^{\complement}\right)\right]-\sum_{j \in S} i_{\phi}^{\mathrm{w}}\left[\underline{X}\left(S_{j}\right): \underline{X}\left(\left(S_{j}\right)^{\complement}\right)\right] } \\
& =\sum_{j \in S}\left\{i_{\phi}^{\mathrm{w}}\left[\left(\underline{X}\left(S_{j}\right), X_{j}\right): \underline{X}\left(S^{\complement}\right)\right]-i_{\phi}^{\mathrm{w}}\left[\underline{X}\left(S_{j}\right):\left(\underline{X}\left(S^{\complement}\right), X_{j}\right)\right]\right\} .
\end{aligned}
$$

After direct computations, we obtain:

$$
i_{\phi}^{\mathrm{w}}\left[\left(\underline{X}\left(S_{j}\right), X_{j}\right): \underline{X}\left(S^{\complement}\right)\right]=i_{\psi\left(S_{j} \cup S^{\complement}\right)}^{\mathrm{w}}\left[\underline{X}\left(S_{j}\right): \underline{X}\left(S^{\complement}\right)\right]+i_{\phi}^{\mathrm{w}}\left[X_{j}: \underline{X}\left(S^{\complement}\right) \mid \underline{X}\left(S_{j}\right)\right],
$$

and

$$
i_{\phi}^{\mathrm{w}}\left[\underline{X}\left(S_{j}\right):\left(\underline{X}\left(S^{\complement}\right), X_{j}\right)\right]=i_{\psi\left(S_{j} \cup S^{\complement}\right)}^{\mathrm{w}}\left[\underline{X}\left(S_{j}\right): \underline{X}\left(S^{\complement}\right)\right]+i_{\phi}^{\mathrm{w}}\left[X_{j}: \underline{X}\left(S_{j}\right) \mid \underline{X}\left(S^{\complement}\right)\right] .
$$

Here $i_{\phi}^{\mathrm{w}}\left[X_{j}: \underline{X}\left(S^{\complement}\right) \mid \underline{X}\left(S_{j}\right)\right], i_{\phi}^{\mathrm{w}}\left[X_{j}: \underline{X}\left(S_{j}\right) \underline{X}\left(S^{\complement}\right)\right]$ are mutual-conditional WEs emerging as in the proof of Theorem 3 from [5]:

$$
\begin{aligned}
i_{\phi}^{\mathrm{w}}\left[X_{j}: \underline{X}\left(S^{\complement}\right) \mid \underline{X}\left(S_{j}\right)\right] & =\mathbb{E}\left(\phi(\underline{X}) \log \frac{f\left(X_{j}, \underline{X}\left(S^{\complement}\right) \mid \underline{X}\left(S_{j}\right)\right)}{f\left(X_{j} \mid \underline{X}\left(S_{j}\right)\right) f\left(\underline{X}\left(S^{\complement}\right) \mid \underline{X}\left(S_{j}\right)\right)}\right) \\
& =\int_{\mathcal{X}_{1}^{n}} \phi(\underline{x}) f(\underline{x}) \log \frac{f\left(x_{j}, \underline{x}\left(S^{\complement}\right) \mid \underline{x}\left(S_{j}\right)\right)}{f\left(x_{j} \mid \underline{x}\left(S_{j}\right)\right) f\left(\underline{x}\left(S^{\complement}\right) \mid \underline{x}\left(S_{j}\right)\right)} \mathrm{d} \underline{x}, \\
i_{\phi}^{\mathrm{w}}\left[X_{j}: \underline{X}\left(S_{j}\right) \mid \underline{X}\left(S^{\complement}\right)\right] & =\mathbb{E}\left(\phi(\underline{X}) \log \frac{f\left(X_{j}, \underline{X}\left(S_{j}\right) \mid \underline{X}\left(S^{\complement}\right)\right)}{f\left(X_{j} \mid \underline{X}\left(S^{\complement}\right)\right) f\left(\underline{X}\left(S_{j}\right) \mid \underline{X}\left(S^{\complement}\right)\right)}\right) \\
& =\int_{\mathcal{X}_{1}^{n}} \phi(\underline{x}) f(\underline{x}) \log \frac{f\left(x_{j}, \underline{x}\left(S_{j}\right) \mid \underline{x}\left(S^{\complement}\right)\right)}{f\left(x_{j} \mid \underline{x}\left(S^{\complement}\right)\right) f\left(\underline{x}\left(S_{j}\right) \mid \underline{x}\left(S^{\complement}\right)\right)} \mathrm{d} \underline{x} .
\end{aligned}
$$

In the remaining argument we will make an extensive use of definition (2.5), employing WF $\psi(S)$ for a number of choices of set $S$. 
Using mutual-conditional WEs we can write:

$$
\begin{aligned}
k i_{\phi}^{\mathrm{w}} & {\left[\underline{X}(S): \underline{X}\left(S^{\complement}\right)\right]-\sum_{j \in S} i_{\phi}^{\mathrm{w}}\left[\underline{X}\left(S_{j}\right): \underline{X}\left(\left(S_{j}\right)^{\complement}\right)\right] } \\
= & \sum_{j \in S}\left\{i_{\phi}^{\mathrm{w}}\left[X_{j}: \underline{X}\left(S^{\complement}\right) \mid \underline{X}\left(S_{j}\right)\right]-i_{\phi}^{\mathrm{w}}\left[X_{j}: \underline{X}\left(S_{j}\right) \mid \underline{X}\left(S^{\complement}\right)\right]\right\} \\
= & \sum_{j \in S}\left[h_{\psi(S)}^{\mathrm{w}}\left(X_{j} \mid \underline{X}\left(S_{j}\right)\right)-h_{\phi}^{\mathrm{w}}\left(X_{j} \mid \underline{X}\left(S^{\complement}\right), \underline{X}\left(S_{j}\right)\right)\right. \\
& \left.\quad-h_{\psi\left(j \cup S^{\complement}\right)}^{\mathrm{w}}\left(X_{j} \mid \underline{X}\left(S^{\complement}\right)\right)-h_{\phi}^{\mathrm{w}}\left(X_{j} \mid \underline{X}\left(S^{\complement}\right), \underline{X}\left(S_{j}\right)\right)\right] \\
= & \sum_{j \in S}\left[h_{\psi(S)}^{\mathrm{w}}\left(X_{j} \mid \underline{X}\left(S_{j}\right)\right)-h_{\psi\left(j \cup S^{\complement}\right)}^{\mathrm{w}}\left(X_{j} \mid \underline{x}\left(S^{\complement}\right)\right)\right] .
\end{aligned}
$$

Summing over all subsets of size $k$ and reversing the order of summation, we obtain:

$$
\begin{aligned}
& \sum_{S \subseteq I: \#(S)=k}\left\{k i_{\phi}^{\mathrm{w}}\left[\underline{X}(S): \underline{X}\left(S^{\complement}\right)\right]-\sum_{j \in S} i_{\phi}^{\mathrm{w}}\left[\underline{X}\left(S_{j}\right): \underline{X}\left(\left(S_{j}\right)^{\complement}\right)\right]\right\} \\
&= \sum_{j=1}^{n} \sum_{S \subseteq I: \#(S)=k, j \in S}\left[\left[h_{\psi\left(j \cup S_{j}\right)}^{\mathrm{w}}\left(X_{j} \mid \underline{X}\left(S_{j}\right)\right)-h_{\psi\left(j \cup S^{\complement}\right)}^{\mathrm{w}}\left(X_{j} \mid \underline{X}\left(S^{\complement}\right)\right)\right] .\right.
\end{aligned}
$$

The RHS of (2.26) can be rewritten in the following way:

$$
\sum_{j=1}^{n} \sum_{S^{\prime}: \#\left(S^{\prime}\right)=k-1, j \notin S}\left[h_{\psi\left(S^{\prime} \cup j\right)}^{\mathrm{w}}\left(X_{j} \mid \underline{X}\left(S^{\prime}\right)\right)-h_{\psi\left(\left\{S^{\prime} \cup j\right\}^{\complement} \cup j\right)}^{\mathrm{w}}\left(X_{j} \mid \underline{X}\left(\left\{S^{\prime} \cup j\right\}^{\complement}\right)\right)\right],
$$

or equivalently

$$
\sum_{j=1}^{n}\left[\sum_{S^{\prime}: \#\left(S^{\prime}\right)=k-1, S^{\prime} \subset\{j\}^{\complement}} h_{\psi\left(S^{\prime} \cup j\right)}^{\mathrm{w}}\left(X_{j} \mid \underline{X}(S)\right)-\sum_{S^{\prime \prime}: \#\left(S^{\prime \prime}\right)=n-k, S^{\prime \prime} \subset\{j\}^{\complement}} h_{\psi\left(S^{\prime \prime} \cup j\right)}^{\mathrm{w}}\left(X_{j} \mid \underline{X}\left(S^{\prime \prime}\right)\right)\right] .
$$

Since $k \leq\lfloor n / 2\rfloor$, then $k-1<n-k$. A set $S^{\prime \prime}$ with $n-k$ elements has $\left(\begin{array}{l}n-1 \\ k-1\end{array}\right)$ subsets of size $k-1$. Owing to Lemma 1.3 from [9], for each such subset $\widetilde{S} \subset S^{\prime \prime}$, under assumption (2.20) we have that

$$
h_{\psi\left(S^{\prime \prime} \cup j\right)}^{\mathrm{w}}\left(X_{j} \mid \underline{X}\left(S^{\prime \prime}\right)\right) \leq h_{\psi(\widetilde{S} \cup j)}^{\mathrm{w}}\left(X_{j} \mid \underline{X}(\widetilde{S})\right) .
$$

With the same argument as in [5] we conclude from (2.27) that

$$
\sum_{S \subseteq I: \#(S)=k}\left\{k i_{\phi}^{\mathrm{w}}\left[\underline{X}(S): \underline{X}\left(S^{\complement}\right)\right]-\sum_{j \in S} i_{\phi}^{\mathrm{w}}\left[\underline{X}\left(S_{j}\right): \underline{X}\left(\left(S_{j}\right)^{\complement}\right)\right]\right\} \geq 0 .
$$

Then, since each set of size $k$ occurs $n-k+1$ times in the second sum, we can write

$$
k \sum_{S \subseteq I: \#(S)=k} i_{\phi}^{\mathrm{w}}\left(\underline{X}(S): \underline{X}\left(S^{\complement}\right)\right) \geq(n-k+1) \sum_{S^{\prime} \subseteq I: \#\left(S^{\prime}\right)=k-1} i_{\phi}^{\mathrm{w}}\left(\underline{X}\left(S^{\prime}\right): \underline{X}\left(S^{\prime}\right)\right) .
$$

Dividing by $k\left(\begin{array}{l}n \\ k\end{array}\right)$ concludes the proof. 


\section{Gaussian weighted entropies}

As we said in the introduction, the WDIs are connected with the Gaussian WE $h_{\phi}^{\mathrm{w}}\left(f_{\mathbf{C}}^{\mathrm{No}}\right):=$ $-\int_{\mathbb{R}^{d}} \phi(\mathbf{x}) f_{\mathbf{C}}^{\mathrm{No}}(\mathbf{x}) \log f_{\mathbf{C}}^{\mathrm{No}}(\mathbf{x}) \mathrm{d} \mathbf{x}$; cf. (1.3), (1.5) . Throughout the paper we use a number of properties established in [9]. One of them is maximization of the $\mathrm{WE} h_{\phi}^{\mathrm{w}}(f):=-\int_{\mathbb{R}^{d}} \phi(\mathbf{x}) f(\mathbf{x}) \log f(\mathbf{x}) \mathrm{d} \mathbf{x}$ at $f=f_{\mathbf{C}}^{\text {No }}$. More precisely, consider the following inequalities

$$
\begin{gathered}
\int_{\mathbb{R}^{d}} \phi(\mathbf{x})\left[f(\mathbf{x})-f_{\mathbf{C}}^{\mathrm{No}}(\mathbf{x})\right] \mathrm{d} \mathbf{x} \geq 0 \\
\log \left[(2 \pi)^{d}(\operatorname{det} \mathbf{C})\right] \int_{\mathbb{R}^{d}} \phi(\mathbf{x})\left[f(\mathbf{x})-f_{\mathbf{C}}^{\mathrm{No}}(\mathbf{x})\right] \mathrm{d} \mathbf{x}+\operatorname{tr}\left[\mathbf{C}^{-1}\left(\mathbf{\Phi}_{\mathbf{C}}^{\mathrm{No}}-\mathbf{\Phi}\right)\right] \leq 0 .
\end{gathered}
$$

Theorem 3.1 Let $\mathbf{X}=\mathbf{X}_{1}^{d} \sim f(\mathbf{x}), \mathbf{x} \in \mathbb{R}^{d}$, be a random vector with PDF $f$, mean zero and covariance matrix

$$
\mathbf{C}=\mathbb{E}_{\mathbf{C}}\left(\left(\mathbf{X}_{1}^{d}\right)\left(\mathbf{X}_{1}^{d}\right)^{\mathrm{T}}\right)=\int_{\mathbb{R}^{d}} \mathbf{x x}^{\mathrm{T}} f_{\mathbf{C}}^{\mathrm{No}}(\mathbf{x}) \mathrm{d} \mathbf{x} .
$$

Set:

$$
\mathbf{\Phi}=\mathbb{E}_{\mathbf{C}}\left(\left(\mathbf{X}_{1}^{d}\right)\left(\mathbf{X}_{1}^{d}\right)^{\mathrm{T}} \phi\left(\mathbf{X}_{1}^{d}\right)\right)=\int_{\mathbb{R}^{d}} \mathbf{x} \mathbf{x}^{\mathrm{T}} \phi(\mathbf{x}) f_{\mathbf{C}}^{\mathrm{No}}(\mathbf{x}) \mathrm{d} \mathbf{x}
$$

and suppose that (3.1) is fulfilled. Then

$$
h_{\phi}^{\mathrm{w}}(f) \leq h_{\phi}^{\mathrm{w}}\left(f_{\mathbf{C}}^{\mathrm{No}}\right)
$$

with equality iff $f=f_{\mathbf{C}}^{\mathrm{No}}$ modulo $\phi$.

The proof of Theorem 3.1 follows the argument in Example 3.1 from [9] repeated verbatim in the multi-dimensional setting.

A conditional form of Theorem 3.1 is Theorem 3.2 below. The corresponding assertion for the standard entropy was noted in an earlier literature. See, e.g., Ref. [6, P. 1516]: the proof of Theorem 29, item (c), the reference to a conditional version of [6, Lemma 5]. The proof of Theorem 3.2 is essentially hinted in its statement (see Eqn (3.6) ), and we omit it from the paper.

Given a $d \times d$ positive-definite matrix $\mathbf{C}$ and $p=1, \ldots, d-1$, write $\mathbf{C}$ in the block form:

$$
\mathbf{C}=\left(\begin{array}{cc}
\mathbf{C}_{1}^{p} & \mathbf{C}_{n-p}^{p} \\
\mathbf{C}_{p}^{n-p} & \mathbf{C}_{p+1}^{d}
\end{array}\right)
$$

where $\mathbf{C}_{n-p}^{p}$ and $\mathbf{C}_{p}^{n-p}$ are mutually transposed $p \times(n-p)$ and $(n-p) \times p$ matrices. Given $\mathbf{x}=\left(\begin{array}{c}\mathbf{x}_{1}^{p} \\ \mathbf{x}_{p+1}^{d}\end{array}\right)$, set $\mathbf{D} \mathbf{x}_{p+1}^{d}=\mathbf{C}_{p}^{n-p}\left(\mathbf{C}_{p+1}^{d}\right)^{-1} \mathbf{x}_{p+1}^{d}$ and $\mathbf{K}_{1}^{p}=\mathbf{C}_{1}^{p}-\mathbf{C}_{p}^{n-p}\left(\mathbf{C}_{p+1}^{d}\right)^{-1} \mathbf{C}_{n-p}^{p}$. Correspondingly, if $\mathbf{X}=\mathbf{X}_{1}^{d}$ is a random vector (RV) with PDF $f_{\mathbf{X}}$ and covariance matrix $\mathbf{C}$ then $\mathbf{C}_{1}^{p}$ represents the covariance matrix for vector $\mathbf{X}_{1}^{p}$, with $\operatorname{PDF} f_{\mathbf{X}_{1}^{p}}\left(\mathbf{x}_{1}^{p}\right)$. Let $\mathbf{X}_{p+1}^{d}$ stand for the residual/remaining random vector and set $f_{\mathbf{X}_{p+1}^{d} \mid \mathbf{X}_{1}^{p}}\left(\mathbf{x}_{p+1}^{d} \mid \mathbf{x}_{1}^{p}\right)=\frac{f_{\mathbf{X}}\left(\mathbf{x}_{1}^{d}\right)}{f_{\mathbf{X}_{1}^{p}}\left(\mathbf{x}_{1}^{p}\right)}$. Also denote by $\mathbf{N}, \mathbf{N}_{1}^{p}$ 
and $\mathbf{N}_{p+1}^{d}$ the corresponding Gaussian vectors, with PDFs $f_{\mathbf{N}}(\mathbf{x})=f_{\mathbf{C}}^{\mathrm{No}}(\mathbf{x}), f_{\mathbf{N}_{1}^{p}}\left(\mathbf{x}_{1}^{p}\right)=f_{\mathbf{C}_{1}^{p}}^{\mathrm{No}}\left(\mathbf{x}_{1}^{p}\right)$ and $f_{\mathbf{N}_{p+1}^{d}}^{\text {No }} \mid \mathbf{N}_{1}^{p}\left(\mathbf{x}_{p+1}^{d} \mid \mathbf{x}_{1}^{p}\right)$. Finally, for a given $\mathrm{WF} \mathbf{x} \in \mathbb{R}^{d} \mapsto \phi(\mathbf{x})$ set:

$$
\begin{gathered}
\psi\left(\mathbf{x}_{1}^{p}\right)=\int_{\mathbb{R}^{n-p}} \phi(\mathbf{x}) f_{\mathbf{N}_{p+1}^{d} \mid \mathbf{N}_{1}^{p}}\left(\mathbf{x}_{p+1}^{d} \mid \mathbf{x}_{1}^{p}\right) \mathrm{d} \mathbf{x}_{p+1}^{d}, \\
\alpha\left(\mathbf{C}_{1}^{p}\right)=\int_{\mathbb{R}^{p}} \psi\left(\mathbf{x}_{1}^{p}\right) f_{\mathbf{N}_{1}^{p}}\left(\mathbf{x}_{1}^{p}\right) \mathrm{d} \mathbf{x}_{1}^{p}, \alpha(\mathbf{C})=\int_{\mathbb{R}^{d}} \phi(\mathbf{x}) f_{\mathbf{N}}(\mathbf{x}) \mathrm{d} \mathbf{x} \\
\mathbf{\Psi}_{\mathbf{N}_{1}^{p}}=\int_{\mathbb{R}^{p}}\left[\mathbf{x}_{1}^{p}\left(\mathbf{x}_{1}^{p}\right)^{\mathrm{T}}\right] \psi\left(\mathbf{x}_{1}^{p}\right) f_{\mathbf{N}_{1}^{p}}\left(\mathbf{x}_{1}^{p}\right) \mathrm{d} \mathbf{x}_{1}^{p}, \mathbf{\Phi}_{\mathbf{N}}=\int_{\mathbb{R}^{d}}\left(\mathbf{x x}^{\mathrm{T}}\right) \phi(\mathbf{x}) f_{\mathbf{N}_{1}^{d}}(\mathbf{x}) \mathrm{d} \mathbf{x} .
\end{gathered}
$$

Also, consider inequalities

$$
\begin{gathered}
\int_{\mathbb{R}^{d}} \phi(\mathbf{x}) f_{\mathbf{X}_{1}^{p}}\left(\mathbf{x}_{1}^{p}\right)\left[f_{\mathbf{X}_{p+1}^{d} \mid \mathbf{X}_{1}^{p}}\left(\mathbf{x}_{p+1}^{d} \mid \mathbf{x}_{1}^{p}\right)-f_{\mathbf{N}_{p+1}^{d} \mid \mathbf{N}_{1}^{p}}\left(\mathbf{x}_{p+1}^{d} \mid \mathbf{x}_{1}^{p}\right)\right] \mathrm{d} \mathbf{x} \geq 0, \\
\int_{\mathbb{R}^{d}} \phi(\mathbf{x})\left[f_{\mathbf{X}}(\mathbf{x})-f_{\mathbf{N}}(\mathbf{x})\right]\left\{\log \left[(2 \pi)^{p} \operatorname{det}\left(\mathbf{K}_{1}^{p}\right)^{-1}\right]\right. \\
\left.+(\log e)\left[\left(\mathbf{x}_{1}^{p}-\mathbf{D} \mathbf{x}_{p+1}^{d}\right)^{\mathrm{T}}\left(\mathbf{K}_{1}^{p}\right)^{-1}\left(\mathbf{x}_{1}^{p}-\mathbf{D} \mathbf{x}_{p+1}^{d}\right)\right]\right\} \mathrm{d} \mathbf{x} \leq 0 .
\end{gathered}
$$

Theorem 3.2 Make an assumption that bounds (3.5) are satisfied. Then the following inequality holds true:

$$
\begin{gathered}
h_{\phi}^{\mathrm{w}}\left(\mathbf{X}_{p+1}^{d} \mid \mathbf{X}_{1}^{p}\right):=-\int_{\mathbb{R}^{d}} \phi(\mathbf{x}) f_{\mathbf{X}}(\mathbf{x}) \log f_{\mathbf{X}_{p+1}^{d} \mid \mathbf{X}_{1}^{p}\left(\mathbf{x}_{p+1}^{d} \mid \mathbf{x}_{1}^{p}\right) \mathrm{d} \mathbf{x}} \\
\leq h_{\phi}^{\mathrm{w}}\left(\mathbf{N}_{p+1}^{d} \mid \mathbf{N}_{1}^{p}\right)=h_{\phi}^{\mathrm{w}}(\mathbf{N})-h_{\psi}^{\mathrm{w}}\left(\mathbf{N}_{1}^{p}\right) \\
=\frac{\alpha(\mathbf{C})}{2} \log \left[(2 \pi)^{d} \operatorname{det} \mathbf{C}\right]+\frac{\log e}{2} \operatorname{tr}\left[\mathbf{C}^{-1} \mathbf{\Phi}_{\mathbf{N}}\right] \\
\quad-\frac{\alpha\left(\mathbf{C}_{1}^{p}\right)}{2} \log \left[(2 \pi)^{p} \operatorname{det} \mathbf{C}_{1}^{p}\right]-\frac{\log e}{2} \operatorname{tr}\left[\left(\mathbf{C}_{1}^{p}\right)^{-1} \mathbf{\Psi}_{\left.\mathbf{N}_{1}^{p}\right] .}\right.
\end{gathered}
$$

\section{Weighted entropies under mappings}

In this section we give a series general theorems (Theorems 4.1-4.3 and Theorem 4.4) reflecting properties of the WEs under mappings of random variables (an example is a sum). Of a special importance for us is Theorem 4.3 used in Section 5. In essence, Theorems 4.1-4.3 are repetitions of their counterparts from [9], and we omit their proofs.

Theorem 4.1 (Cf. Lemma 1.1 from [9].) Let $\left(\mathcal{X}, \mathfrak{X}, \nu_{\mathcal{X}}\right),\left(\mathcal{Y}, \mathfrak{Y}, \nu_{Y}\right)$ be a pair of Lebesgue spaces and suppose $X, Y$ are random elements in $(\mathcal{X}, \mathfrak{X}),(\mathcal{Y}, \mathfrak{Y})$ and $P M / D F s f_{X}, f_{Y}$, relative to measures $\nu_{\mathcal{X}}, \nu_{\mathcal{Y}}$, respectively. Suppose $\eta:(\mathcal{X}, \mathfrak{X}) \rightarrow(\mathcal{Y}, \mathfrak{Y})$ is a measurable map onto, and that $\nu_{\mathcal{Y}}(B)=\nu_{\mathcal{X}}\left(\eta^{-1} B\right), B \in \mathfrak{Y}$. Consider the partition of $\mathcal{X}$ with elements $\mathcal{B}(y):=\{x \in$ 
$\mathcal{X}: \eta x=y\}$ and let $\nu_{\mathcal{X}}(\cdot \mid y)$ be the family of induced measures on $\mathcal{B}(y), y \in \mathcal{Y}$. Suppose that $f_{Y}(y)=\int_{\mathcal{B}(y)} f_{X}(x) \nu(\mathrm{d} x \mid y)$ and for $x \in \mathcal{B}(y)$ let $f_{X \mid Y}(x \mid y):=\frac{f_{X}(x)}{f_{Y}(y)}$ denote the $P M / D F$ of $X$ conditional on $Y=y$. (Recall, $f_{X \mid Y}(\cdot \mid y)$ is a family of PM/DFs defined for $f_{Y-a . a}$ $y \in \mathcal{Y}$ such that $\int_{\mathcal{X}} G(x) f_{X}(x) \nu_{\mathcal{X}}(\mathrm{d} x)=\int_{\mathcal{Y}} \int_{\mathcal{B}(y)} G(x) f_{X \mid Y}(x \mid y) \nu_{\mathcal{X}}(\mathrm{d} x \mid y) f_{Y}(y) \nu \mathcal{Y}(\mathrm{d} y)$ for any nonnegative measurable function $G$.) Suppose that a $W F \quad x \in \mathcal{X} \mapsto \phi(x) \geq 0$ obeys

$$
\int_{\mathcal{X}} \phi(x) f_{X}(x)\left[f_{X \mid Y}(x \mid \eta x)-1\right] \nu_{\mathcal{X}}(\mathrm{d} x) \leq 0
$$

and set

$$
\psi(y)=\int_{\mathcal{B}(y)} \phi(x) f_{X \mid Y}(x \mid y) \nu(\mathrm{d} x \mid y), \quad y \in \mathcal{Y} .
$$

Then

$$
\begin{aligned}
& h_{\phi}^{\mathrm{w}}(X) \geq h_{\psi}^{\mathrm{w}}(Y):=-\int_{\mathcal{Y}} \psi(y) f_{Y}(y) \log f_{Y}(y) \nu_{Y}(\mathrm{~d} y), \quad \text { or } \\
& h_{\phi}^{\mathrm{w}}(X \mid Y):=-\int_{\mathcal{X}} \phi(x) f_{X}(x) \log f_{X \mid Y}(x \mid y(x)) \nu_{\mathcal{X}}(\mathrm{d} x) \geq 0,
\end{aligned}
$$

with equality iff $\phi(x)\left[f_{X \mid Y}(x \mid \eta x)-1\right]=0$ for $f$-a.a. $x \in \mathcal{X}$.

In particular, suppose that for $f_{Y}$-a.a. $y \in \mathcal{Y}$ set $\mathcal{B}(y)$ contains at most countably many values and $\nu(\cdot \mid y)$ is a counting measure with $\nu_{1}(x)=1, x \in \mathcal{B}(y)$. Then the value $f_{X \mid Y}(x \mid \eta x)$ yields the conditional probability $\mathbb{P}(X=x \mid Y=\eta x)$, which is $\leq 1$ for $f_{Y}$-a.a. $y \in \mathcal{Y}$. Then $h_{\phi}^{\mathrm{w}}(X \mid Y) \geq 0$ and the bound is strict unless, modulo $\phi$, map $\eta$ is $1-1$.

Theorem 4.2 (Cf. Lemma 1.2 from [9].) Let $\left(\mathcal{X}, \mathfrak{X}, \nu_{\mathcal{X}}\right),\left(\mathcal{Y}, \mathfrak{Y}, \nu_{\mathcal{Y}}\right),\left(\mathcal{Z}, \mathfrak{Z}, \nu_{\mathcal{Z}}\right)$ be a triple of $S M S$ s and suppose $X, Y, Z$ are random elements in $(\mathcal{X}, \mathfrak{X}),(\mathcal{Y}, \mathfrak{Y}),(\mathcal{Z}, \mathfrak{Z})$. Let $f_{X}$ be the $P M / D F$ for $X$ relative to measure $\nu_{\mathcal{X}}$ and $f_{Y, Z}$ the joint $P M / D F$ for $Y, Z$ relative to measures $\nu \mathcal{Y} \times \nu_{\mathcal{Z}}$. Further, set $f_{Z}(z):=\int_{\mathcal{Y}} f(y, z) \nu \mathcal{Y}(\mathrm{d} y)$ and $f_{Y \mid Z}(y \mid z)=\frac{f_{Y, Z}(y, z)}{f_{Z}(z)}$. Suppose that

$$
\eta:(\mathcal{X}, \mathfrak{X}) \rightarrow(\mathcal{Y}, \mathfrak{Y}), \quad \zeta:(\mathcal{X}, \mathfrak{X}) \rightarrow(\mathcal{Z}, \mathfrak{Z})
$$

is a pair of measurable maps onto, and that

$$
\nu_{\mathcal{Y}}(A)=\nu_{\mathcal{X}}\left(\eta^{-1} A\right), A \in \mathfrak{Y}, \quad \nu_{\mathcal{Z}}(B)=\nu_{\mathcal{X}}\left(\zeta^{-1} B\right), B \in \mathfrak{Z} .
$$

Consider the partition of $\mathcal{X}$ with elements $\mathcal{B}(y, z):=\{x \in \mathcal{X}: \eta x=y, \zeta x=z\}$ and let $\nu \mathcal{X}(\cdot \mid y, z)$ be the family of induced measures on $\mathcal{B}(y, z),(y, z) \in \mathcal{Y} \times \mathcal{Z}$. Suppose that

$$
f_{Y, Z}(y, z)=\int_{\mathcal{B}(y, z)} f_{X}(x) \nu_{\mathcal{X}}(\mathrm{d} x \mid y, z)
$$


and for $x \in \mathcal{B}(y, z)$ let $f_{X \mid Y, Z}(x \mid y, z):=\frac{f_{X}(x)}{f_{Y, Z}(y, z)}$ denote the PM/DF of $X$ conditional on $Y=y, Z=z .\left(\right.$ Recall, $f_{X \mid Y, Z}(\cdot \mid y, z)$ is a family of PM/DFs defined for $f_{Y, Z}-a . a(y, z) \in \mathcal{Y} \times \mathcal{Z}$ such that

$$
\left.\int_{\mathcal{X}} G(x) f_{X}(x) \nu \mathcal{X}(\mathrm{d} x)=\int_{\mathcal{Y} \times \mathcal{Z}} \int_{\mathcal{B}(y, z)} G(x) f_{X \mid Y, Z}(x \mid y, z) \nu \mathcal{X}(\mathrm{d} x \mid y, z) f_{Y, Z}(y, z)\right) \nu \mathcal{Y}(\mathrm{d} y) \nu_{\mathcal{Z}}(\mathrm{d} z)
$$

for any non-negative measurable function $G$.) Assume that a WF $x \mapsto \phi(x) \geq 0$ obeys

$$
\int_{\mathcal{X}} \phi(x) f(x)\left[f_{X \mid Y, Z}(x \mid \eta x, \zeta x)-1\right] \nu \mathcal{X}(\mathrm{d} x) \leq 0
$$

and set

$$
\psi(y, z)=\int_{\mathcal{B}(y, z)} \phi(x) f_{X \mid Y, Z}(x \mid y, z) \nu(\mathrm{d} x \mid y, z)
$$

Then

$$
\begin{aligned}
& -\int_{\mathcal{Y} \times \mathcal{Z}} \psi(y, z) f_{Y, Z}(y, z) \log f_{Y \mid Z}(y \mid z) \nu \mathcal{Y}(\mathrm{d} y) \nu_{\mathcal{Z}}(\mathrm{d} z) \\
& =: h_{\psi}^{\mathrm{w}}(Y \mid Z) \leq h_{\phi}^{\mathrm{w}}(X \mid Z):=-\int_{\mathcal{X}} \phi(x) f_{X}(x) \log f_{X \mid Z}(x \mid \zeta x) \nu(\mathrm{d} x)
\end{aligned}
$$

equality iff $\phi(x)\left[f_{X \mid Y, Z}(x \mid \eta x, \zeta x)-1\right]=0$ for $f_{X}$-a.a. $x \in \mathcal{X}$.

As in Theorem 4.1, assume $\mathcal{B}(y, z)$ consists of at most countably many values and $\nu(x \mid y, z)=$ $1, x \in \mathcal{B}(y, z)$ for $f_{Y, Z}$-a.a. $(y, z) \in \mathcal{Y} \times \mathcal{Z}$. Then the value $f_{X \mid Y, Z}(x \mid y, z)$ yields the conditional probability $\mathbb{P}(X=x \mid Y=y, Z=z)$, for $f_{Y, Z^{-}}$a.a. $y, z \in \mathcal{Y} \times \mathcal{Z}$. Then $h_{\phi}^{\mathrm{w}}(X \mid Z) \geq h_{\psi}^{\mathrm{w}}(Y \mid Z)$, with equality iff, modulo $\phi$, the map $x \mapsto(\eta x, \zeta x)$ is $1-1$.

Theorem 4.3 (Cf. Lemma 1.3 from [9].) Let $\left(\mathcal{X}, \mathfrak{X}, \nu_{\mathcal{X}}\right),\left(\mathcal{Y}, \mathfrak{Y}, \nu_{\mathcal{Y}}\right),\left(\mathcal{Z}, \mathfrak{Z}, \nu_{\mathcal{Z}}\right)$ be a triple of $S M S$ s and suppose $X, Y, Z$ are random elements in $(\mathcal{X}, \mathfrak{X}),(\mathcal{Y}, \mathfrak{Y}),(\mathcal{Z}, \mathfrak{Z})$. Let $f_{X, Y}$ be the joint $P M / D F$ for $X, Y$ relative to measure $\nu_{\mathcal{X}} \times \nu_{\mathcal{Y}}$ and set

$$
f_{Y}(y)=\int_{\mathcal{X}} f_{X, Y}(x, y) \nu_{\mathcal{X}}(\mathrm{d} x), \quad f_{X \mid Y}(x \mid y)=\frac{f_{X, Y}(x, y)}{f_{Y}(y)} .
$$

Suppose that

$$
\xi:(\mathcal{Y}, \mathfrak{Y}) \rightarrow(\mathcal{Z}, \mathfrak{Z})
$$

is a measurable maps onto, and that

$$
\nu_{\mathcal{Z}}(C)=\nu_{\mathcal{Y}}\left(\xi^{-1} C\right), C \in \mathfrak{Z} .
$$


Consider a partition of $\mathcal{Y}$ with elements $\mathcal{C}(z):=\{y \in \mathcal{Y}: \xi y=z\}$ and let $\nu \mathcal{Y}(\cdot \mid z)$ be the family of induced measures on $\mathcal{C}(z), z \in \mathcal{Z}$. Given $(x, z) \in \mathcal{X} \times \mathcal{Z}$ and $y \in \mathcal{C}(z)$, let

$$
f_{X, Z}(x, z)=\int_{\mathcal{C}(z)} f_{X, Y}(x, y) \nu \mathcal{Y}(\mathrm{d} y \mid z), f_{Z}(z)=\int_{\mathcal{X}} f_{X, Z}(x, z) \nu \mathcal{X}(\mathrm{d} x),
$$

and

$$
f_{X \mid Z}(x \mid z)=\frac{f_{X, Z}(x, z)}{f_{Z}(z)}, \quad f_{Y \mid Z}(y \mid z)=\frac{f_{Y}(y)}{f_{Z}(z)} .
$$

Assume that a $W F(x, y) \mapsto \phi(x, y) \geq 0$ obeys

$$
\int_{\mathcal{X} \times \mathcal{Y}} \phi(x, y)\left[f_{X, Y}(x, y)-f_{Z}(\xi y) f_{X \mid Z}(x \mid \xi y) f_{Y \mid Z}(y \mid \xi y)\right] \nu \mathcal{X}(\mathrm{d} x) \nu \mathcal{Y}(\mathrm{d} y) \geq 0
$$

and set

$$
\psi(x, z)=\int_{\mathcal{C}(z)} \phi(x, y) f_{Y \mid Z}(y \mid z) \nu \mathcal{Y}(\mathrm{d} y \mid z)
$$

Then

$$
\begin{aligned}
& -\int_{\mathcal{X} \times \mathcal{Z}} \psi(x, z) f_{X, Z}(x, z) \log f_{X \mid Z}(y \mid z) \nu_{\mathcal{X}}(\mathrm{d} x) \nu_{\mathcal{Z}}(\mathrm{d} z) \\
& \quad=: h_{\psi}^{\mathrm{w}}(X \mid Z) \geq h_{\phi}^{\mathrm{w}}(X \mid Y):=-\int_{\mathcal{X} \times \mathcal{Y}} \phi(x, y) f_{X}(x) \log f_{X \mid Y}(x \mid y) \nu_{\mathcal{X}}(\mathrm{d} x) \nu \mathcal{Y}(\mathrm{d} y) .
\end{aligned}
$$

Furthermore, equality in (4.9) holds iff $X$ and $Y$ are conditionally independent given $Z$ modulo $\phi$, i.e. $\phi(x, y)\left[f_{X, Y}(x, y)-f_{Z}(\xi y) f_{X \mid Z}(x \mid \xi y) f_{Y \mid Z}(y \mid \xi y)\right]=0$.

We will use an alternative notation $h_{\phi}^{\mathrm{w}}(\mathbf{X}):=h_{\phi}^{\mathrm{w}}\left(f_{\mathbf{X}}\right)$ where $\mathbf{X}=\mathbf{X}_{1}^{d}=\left(\begin{array}{c}X_{1} \\ \vdots \\ X_{d}\end{array}\right)$ is a $d$ dimensional random vector with $\operatorname{PDF} f_{\mathbf{X}}(\mathbf{x})$. In this context, we employ the notation $\mathbf{X} \sim f_{\mathbf{X}}$, $\mathbf{Y} \sim f_{\mathbf{Y}},(\mathbf{X}, \mathbf{Y}) \sim f_{\mathbf{X}, \mathbf{Y}}$ and $(\mathbf{X} \mid \mathbf{Y}) \sim f_{\mathbf{X} \mid \mathbf{Y}}$ where $f_{\mathbf{X} \mid \mathbf{Y}}(\mathbf{x} \mid \mathbf{y})=\frac{f_{\mathbf{X}, \mathbf{Y}}(\mathbf{x}, \mathbf{y})}{f_{\mathbf{Y}}(\mathbf{y})}$

Theorem 4.4 below mimics a result in [2], extending from the case of a standard entropy to that of the WE. A number of facts are related to the conditional WE

$$
h_{\phi}^{\mathrm{w}}(\mathbf{X} \mid \mathbf{Y}):=-\int_{\mathbb{R}^{d} \times \mathbb{R}^{d}} \phi(\mathbf{x}, \mathbf{y}) f_{\mathbf{X}, \mathbf{Y}}(\mathbf{x}, \mathbf{y}) \log f_{\mathbf{X} \mid \mathbf{Y}}(\mathbf{x} \mid \mathbf{y}) \mathrm{d} \mathbf{x} \mathrm{d} \mathbf{y}
$$

or, more generally,

$$
h_{\widetilde{\phi}}^{\mathrm{w}}(\mathbf{U} \mid \mathbf{V}):=-\int_{\mathbb{R}^{d} \times \mathbb{R}^{d}} \widetilde{\phi}(\mathbf{u}, \mathbf{v}) f_{\mathbf{U}, \mathbf{V}}(\mathbf{u}, \mathbf{v}) \log f_{\mathbf{U} \mid \mathbf{V}}(\mathbf{u} \mid \mathbf{v}) \mathrm{d} \mathbf{u} \mathrm{d} \mathbf{v}
$$

Here a pair $(\mathbf{U}, \mathbf{V})$ is a function of $(\mathbf{X}, \mathbf{Y})$ with a joint PM/DF $f_{\mathbf{U}, \mathbf{V}}$, marginal PM/DFs $f_{\mathbf{U}}$, $f_{\mathbf{V}}$ and conditional PM/DF $f_{\mathbf{U} \mid \mathbf{V}}(\mathbf{u} \mid \mathbf{v}):=\frac{f_{\mathbf{U}, \mathbf{V}}(\mathbf{u}, \mathbf{v})}{f_{\mathbf{V}}(\mathbf{v})}$. (Viz., $\mathbf{U}=\mathbf{Y}, \mathbf{V}=\mathbf{X}+\mathbf{Y}$.) WF $\widetilde{\phi}$ may or may not be involved with the map $(\mathbf{X}, \mathbf{Y}) \mapsto(\mathbf{U}, \mathbf{V})$. 
Theorem 4.4 Suppose $\mathbf{X}$ and $\mathbf{Y}$ are independent random vectors of dimension d, with PDFs $f_{\mathbf{X}}$ and $f_{\mathbf{Y}}$ :

$$
(\mathbf{X}, \mathbf{Y}) \sim f_{\mathbf{X}, \mathbf{Y}} \text { where } f_{\mathbf{X}, \mathbf{Y}}(\mathbf{x}, \mathbf{y})=f_{\mathbf{X}}(\mathbf{x}) f_{\mathbf{Y}}(\mathbf{y}), \quad \mathbf{x}, \mathbf{y} \in \mathbb{R}^{d} .
$$

Assume that WF $(\mathbf{x}, \mathbf{y}) \in \mathbb{R}^{d} \times \mathbb{R}^{d^{\prime}} \rightarrow \phi(\mathbf{x}, \mathbf{y}) \geq 0$ obeys

$$
\int_{\mathbb{R}^{d} \times \mathbb{R}^{d}} \phi(\mathbf{x}, \mathbf{y}) f_{\mathbf{Y}}(\mathbf{y})\left[f_{\mathbf{X}}(\mathbf{x})-f_{\mathbf{X}+\mathbf{Y}}(\mathbf{x}+\mathbf{y})\right] \mathrm{d} \mathbf{x} \mathrm{d} \mathbf{y} \geq 0
$$

and set

$$
\theta(\mathbf{v})=\int_{\mathbb{R}^{d}} \phi(\mathbf{v}-\mathbf{y}, \mathbf{y}) f_{\mathbf{Y} \mid \mathbf{X}+\mathbf{Y}}(\mathbf{y} \mid \mathbf{v}) \mathrm{d} \mathbf{y}, \theta^{*}(\mathbf{x})=\int_{\mathbb{R}^{d}} \phi(\mathbf{x}+\mathbf{y}, \mathbf{y}) f_{\mathbf{Y}}(\mathbf{y}) \mathrm{d} \mathbf{y}, \quad \mathbf{v}, \mathbf{x} \in \mathbb{R}^{d} .
$$

Then

$$
h_{\theta}^{\mathrm{w}}(\mathbf{X}+\mathbf{Y}) \geq h_{\theta^{*}}^{\mathrm{w}}(\mathbf{X})
$$

with equality iff $\phi(\mathbf{x}, \mathbf{y}) f_{\mathbf{Y}}(\mathbf{y})\left[f_{\mathbf{X}}(\mathbf{x})-f_{\mathbf{X}+\mathbf{Y}}(\mathbf{x}+\mathbf{y})\right]=0$ for Lebesgue-a.a. $(\mathbf{x}, \mathbf{y}) \in \mathbb{R}^{d} \times \mathbb{R}^{d}$.

Proof. Set: $\phi^{*}(\mathbf{x}, \mathbf{y})=\phi(\mathbf{x}+\mathbf{y}, \mathbf{y})$. The following relations (a)-(c) hold true:

(a) $h_{\theta}^{\mathrm{w}}(\mathbf{X}+\mathbf{Y}) \geq h_{\phi}^{\mathrm{w}}(\mathbf{X}+\mathbf{Y} \mid \mathbf{Y})$,

$$
\text { (b) } h_{\phi}^{\mathrm{w}}(\mathbf{X}+\mathbf{Y} \mid \mathbf{Y})=h_{\phi^{*}}^{\mathrm{w}}(\mathbf{X} \mid \mathbf{Y}), \quad(\mathrm{c}) \quad h_{\phi^{*}}^{\mathrm{w}}(\mathbf{X} \mid \mathbf{Y})=h_{\theta^{*}}^{\mathrm{w}}(\mathbf{X}) \text {. }
$$

Here bound (a) comes from the sub-additivity of the WE, see [9], Theorem 1.3 or Eqn (1.31) from [9]. Next, (b) is derived by applying the following equations:

$$
\begin{aligned}
h_{\phi}^{\mathrm{w}}(\mathbf{X}+\mathbf{Y} \mid \mathbf{Y}) & =\int_{\mathbb{R}^{d}} f_{\mathbf{Y}}(\mathbf{y}) h_{\phi}^{\mathrm{w}}(\mathbf{X}+\mathbf{Y} \mid \mathbf{Y}=\mathbf{y}) \mathrm{d} \mathbf{y} \\
& =-\int_{\mathbb{R}^{d} \times \mathbb{R}^{d}} \phi(\mathbf{x}+\mathbf{y}, \mathbf{y}) f_{\mathbf{Y}}(\mathbf{y}) f_{\mathbf{X} \mid \mathbf{Y}}(\mathbf{x} \mid \mathbf{y}) \log f_{\mathbf{X} \mid \mathbf{Y}}(\mathbf{x} \mid \mathbf{y}) \mathrm{d} \mathbf{x} \mathrm{d} \mathbf{y} .
\end{aligned}
$$

Finally, Eqn (c) holds because $\mathbf{X}$ and $\mathbf{Y}$ are independent.

The proof of Theorem 4.4 is completed by observing that

$$
\begin{aligned}
h_{\phi^{*}}^{\mathrm{w}}(\mathbf{X} \mid \mathbf{Y}) & \left.=-\int_{\mathbb{R}^{d} \times \mathbb{R}^{d}} \phi(\mathbf{x}+\mathbf{y}, \mathbf{y}) f_{\mathbf{X}, \mathbf{Y}}(\mathbf{x}, \mathbf{y}) \log f_{\mathbf{X} \mid \mathbf{Y}}(\mathbf{x} \mid \mathbf{y}) \mathrm{d} \mathbf{x}\right) \mathrm{d} \mathbf{y} \\
& =-\int_{\mathbb{R}^{d}}\left[\int_{\mathbb{R}^{d}} \phi(\mathbf{x}+\mathbf{y}, \mathbf{y}) f_{\mathbf{Y}}(\mathbf{y}) \mathrm{d} \mathbf{y}\right] f_{\mathbf{X}}(\mathbf{x}) \log f_{\mathbf{X}}(\mathbf{x}) \mathrm{d} \mathbf{x} .
\end{aligned}
$$

Remark 4.5 The assertion of Theorem 4.4 remains valid, mutates mutandis, when $\mathbf{X}$ and $\mathbf{Y}$ have different dimensions. Viz., we can assume that $\mathbf{Y}$ has dimension $d^{\prime}<d$ and append $\mathbf{Y}$ and $\mathbf{y}$ with zero entries when we $\operatorname{sum} \mathbf{X}+\mathbf{Y}$ and $\mathbf{x}+\mathbf{y}$. 


\section{Miscellaneous weighted determinant inequalities}

In this section we present a host of WDIs derived from properties of the WEs. As we said before, the proposed inequalities hold when WF $\phi \equiv 1$ (in this case the stated conditions are trivially fulfilled). To stress parallels with 'standard' DIs, we provide references to [2] or [5] in each case under consideration.

Theorem 5.1 (Cf. 2] Theorem 2.) Let $\mathbf{X}, \mathbf{Y}$ be independent d-variate normal vectors with zero means and covariance matrices $\mathbf{C}_{1}, \mathbf{C}_{2}$, respectively: $f_{\mathbf{X}, \mathbf{Y}}(\mathbf{x}, \mathbf{y})=f_{\mathbf{X}}(\mathbf{x}) f_{\mathbf{Y}}(\mathbf{y}), \mathbf{x}, \mathbf{y} \in \mathbb{R}^{d}$, where $f_{\mathbf{X}}=f_{\mathbf{C}_{1}}^{\mathrm{No}}, f_{\mathbf{Y}}=f_{\mathbf{C}_{2}}^{\mathrm{No}}$. Given a WF $(\mathbf{x}, \mathbf{y}) \in \mathbb{R}^{d} \times \mathbb{R}^{d} \mapsto \phi(\mathbf{x}, \mathbf{y}) \geq 0$, positive on an open domain in $\mathbb{R}^{d} \times \mathbb{R}^{d}$, consider a quantity $\beta$ and $d \times d$ matrices $\boldsymbol{\Theta}, \boldsymbol{\Theta}^{*}$ :

$$
\beta=\int_{\mathbb{R}^{d}} \theta(\mathbf{x}) f_{\mathbf{C}_{1}+\mathbf{C}_{2}}^{\mathrm{No}}(\mathbf{x}) \mathrm{d} \mathbf{x}, \boldsymbol{\Theta}=\int_{\mathbb{R}^{d}} \mathbf{x x}^{\mathrm{T}} \theta(\mathbf{x}) f_{\mathbf{C}_{1}+\mathbf{C}_{2}}^{\mathrm{No}}(\mathbf{x}) \mathrm{d} \mathbf{x}, \boldsymbol{\Theta}^{*}=\int_{\mathbb{R}^{d}} \mathbf{x x}^{\mathrm{T}} \theta^{*}(\mathbf{x}) f_{\mathbf{C}_{1}}^{\mathrm{No}}(\mathbf{x}) \mathrm{d} \mathbf{x}
$$

where $\theta$ and $\theta^{*}$ are as in (4.11):

$$
\theta(\mathbf{x})=\int_{\mathbb{R}^{d}} \phi(\mathbf{z}, \mathbf{x}-\mathbf{z}) f_{\mathbf{Y} \mid \mathbf{X}+\mathbf{Y}}(\mathbf{x}-\mathbf{z} \mid \mathbf{x}) \mathrm{d} \mathbf{z}, \quad \theta^{*}(\mathbf{x})=\int_{\mathbb{R}^{d}} \phi(\mathbf{x}+\mathbf{y}, \mathbf{y}) f_{\mathbf{Y}}(\mathbf{y}) \mathrm{d} \mathbf{y} .
$$

Assume the condition emulating (4.10):

$$
\int_{\mathbb{R}^{d} \times \mathbb{R}^{d}} \phi(\mathbf{x}, \mathbf{y}) f_{\mathbf{C}_{2}}^{\mathrm{No}}(\mathbf{y})\left[f_{\mathbf{C}_{1}}^{\mathrm{No}}(\mathbf{x})-f_{\mathbf{C}_{1}+\mathbf{C}_{2}}^{\mathrm{No}}(\mathbf{x}+\mathbf{y})\right] \mathrm{d} \mathbf{x} \mathrm{d} \mathbf{y} \geq 0 .
$$

Then

$$
\beta \log \left[\frac{\operatorname{det}\left(\mathbf{C}_{1}+\mathbf{C}_{2}\right)}{\operatorname{det} \mathbf{C}_{1}}\right]+(\log e)\left\{\operatorname{tr}\left[\left(\mathbf{C}_{1}+\mathbf{C}_{2}\right)^{-1} \mathbf{\Theta}\right]-\operatorname{tr}\left(\mathbf{C}_{1}^{-1} \mathbf{\Theta}^{*}\right)\right\} \geq 0 .
$$

Proof. Using Theorem 4.4 and Eqn (1.3), we can write:

$$
\begin{aligned}
\frac{1}{2} \log \left[(2 \pi)^{d}\left(\operatorname{det}\left(\mathbf{C}_{1}+\mathbf{C}_{2}\right)\right)\right] \int_{\mathbb{R}^{d}} \theta(\mathbf{x}) f_{\mathbf{C}_{1}+\mathbf{C}_{2}}^{\mathrm{No}}(\mathbf{x}) \mathrm{d} \mathbf{x}+\frac{\log e}{2} \operatorname{tr}\left(\mathbf{C}_{1}+\mathbf{C}_{2}\right)^{-1} \mathbf{\Theta} \\
\geq \frac{1}{2} \log \left[(2 \pi)^{d}\left(\operatorname{det} \mathbf{C}_{1}\right)\right] \int_{\mathbb{R}} \theta^{*}(\mathbf{x}) f_{\mathbf{C}_{1}}(\mathbf{x}) \mathrm{d} \mathbf{x}+\frac{\log e}{2} \operatorname{tr} \mathbf{C}_{1}^{-1} \mathbf{\Theta}^{*} .
\end{aligned}
$$

The bound in (5.4) then follows.

Remark 5.2 It is instructive to observe that (5.4) is equivalent to:

$$
\begin{aligned}
\beta \log \left[\operatorname{det}\left(\mathbf{I}+\mathbf{C}_{1}^{-1} \mathbf{C}_{2}\right)\right] \\
+(\log e) \operatorname{tr}\left[\left(\mathbf{C}_{1}+\mathbf{C}_{2}\right)^{-1} \mathbf{\Theta}^{*}-\mathbf{C}_{1}^{-1} \mathbf{\Theta}^{*}+\left(\mathbf{C}_{1}+\mathbf{C}_{2}\right)^{-1} \widetilde{\mathbf{\Theta}}\right] \geq 0
\end{aligned}
$$

where

$$
\widetilde{\boldsymbol{\Theta}}=\int_{\mathbb{R}^{d} \times \mathbb{R}^{d}}\left(\mathbf{x} \mathbf{y}^{\mathrm{T}}+\mathbf{y} \mathbf{x}^{\mathrm{T}}+\mathbf{y} \mathbf{y}^{\mathrm{T}}\right) \phi(\mathbf{x}+\mathbf{y}, \mathbf{y}) f_{\mathbf{C}_{2}}^{\mathrm{No}}(\mathbf{y}) f_{\mathbf{C}_{1}}^{\mathrm{No}}(\mathbf{x}) \mathrm{d} \mathbf{y} \mathrm{d} \mathbf{x} .
$$

This claim is verified by observing that $\boldsymbol{\Theta}=\boldsymbol{\Theta}^{*}+\widetilde{\boldsymbol{\Theta}}$. 
Remark 5.3 As above, we can assume that $\mathbf{C}_{2}$ is a matrix of size $d^{\prime} \times d^{\prime}$, agreeing that in the sum $\mathbf{C}_{1}+\mathbf{C}_{2}$, matrix $\mathbf{C}_{2}$ is identified as a top left block (say). This is possible because in Eqns (5.4) and (5.5) we do not use the inverse $\mathbf{C}_{2}^{-1}$ or the determinant $\operatorname{det} \mathbf{C}_{2}$.

To this end, recall the following theorem from [7:

Theorem 5.4 Let $\mathbf{G}$ and $\mathbf{G}+\mathbf{E}$ be nonsingular matrices where $\mathbf{E}$ is a matrix of rank one. Let $g=\operatorname{tr}\left(\mathbf{E G}^{-1}\right)$. Then $g \neq-1$ and

$$
(\mathbf{G}+\mathbf{E})^{-1}=\mathbf{G}^{-1}-\frac{1}{1+g} \mathbf{G}^{-1} \mathbf{E} \mathbf{G}^{-1} .
$$

The above equation is essentially the Sherman-Morrison formula (see [4], p. 161).

Assuming that $\mathbf{C}_{2}=\mathbf{E}$ has rank 1 and letting $g=\operatorname{tr}\left(\mathbf{E} \mathbf{C}_{1}^{-1}\right)$, inequality (5.4) turns into the following bound:

$$
\left.\beta \log \left[\frac{\operatorname{det}\left(\mathbf{C}_{1}+\mathbf{E}\right)}{\operatorname{det} \mathbf{C}_{1}}\right]+(\log e)\left[-\operatorname{tr}\left(\frac{\mathbf{C}_{1}^{-1} \mathbf{E} \mathbf{C}_{1}}{1+g} \mathbf{\Theta}^{*}\right)+\operatorname{tr}\left\{\left(\mathbf{C}_{1}+\mathbf{E}\right)^{-1} \widetilde{\boldsymbol{\Theta}}\right\}\right)\right] \geq 0 .
$$

The techniques developed so far allows us to prove Theorem 5.5 below rendering a weighted form of Szasz theorem. Suppose $\mathbf{C}$ is a positive definite $d \times d$ matrix. Given $1 \leq k \leq d$ and a set $S \subseteq I^{(d)}:=\{1, \ldots, d\}$ with $\#(S)=k$, denote by $\mathbf{C}(S)$ be the $k \times k$ sub-matrix of $\mathbf{C}$ formed by the rows and columns with indices $i \in S$. With every $S$ we associate a Gaussian random vector $\mathbf{X}(S) \sim f_{\mathbf{C}(S)}^{\mathrm{No}}$ considered as a sub-collection of $\mathbf{X} \sim f_{\mathbf{C}}^{\text {No }}$. Accordingly, conditional PDFs emerge, $f_{S \mid S^{\prime}}^{\mathrm{No}}\left(\mathbf{x}(S) \mid \mathbf{x}\left(S^{\prime}\right)\right)$, for pairs of sets $S, S^{\prime}$ with $S \cap S^{\prime}=\emptyset$, where $\mathbf{x}(S) \in \mathbb{R}^{\#(S)}$, $\mathbf{x}\left(S^{\prime}\right) \in \mathbb{R}^{\#\left(S^{\prime}\right)}$. [The PDF $f_{S \mid S^{\prime}}^{\mathrm{No}}$ is expressed in terms of block sub-matrices forming the inverse matrix $\mathbf{C}\left(S \cup S^{\prime}\right)^{-1}$.]

Further, let a function $\phi(\mathbf{x}) \geq 0, \mathbf{x} \in \mathbb{R}^{d}$, be given, which is positive on an open domain in $\mathbb{R}^{d}$ and set, as in (2.5),

$$
\psi(S ; \mathbf{x}(S))=\int_{\mathbb{R}^{\#\left(S^{\complement}\right)}} \phi(\mathbf{x}) f_{S^{\complement} \mid S}^{\mathrm{No}}\left(\mathbf{x}\left(S^{\complement}\right) \mid \mathbf{x}(S)\right) \mathrm{d} \mathbf{x}\left(S^{\complement}\right) .
$$

Furthermore, define:

$$
\tau(S)=\operatorname{tr}\left[\mathbf{C}(S)^{-1} \mathbf{\Phi}(S)\right], \quad \mathrm{T}(k)=\sum_{S \subseteq I^{(d)}: \#(S)=k} \tau(S)
$$

where matrix $\mathbf{\Phi}(S)$ is given by

$$
\boldsymbol{\Phi}(S)=\boldsymbol{\Phi}(\mathbf{C}(S))=\int_{\mathbb{R} \#(S)} \mathbf{x}(S) \mathbf{x}(S)^{\mathrm{T}} \psi(S ; \mathbf{x}(S)) f_{\mathbf{C}(S)}^{\mathrm{No}}(\mathbf{x}(S)) \mathrm{d} \mathbf{x}(S) .
$$

(For $S=I^{(d)}$, we write simply $\mathbf{\Phi}$; cf. (1.4).) Finally, set:

$$
\alpha(S)=\alpha(\mathbf{C}(S))=\int_{\mathbb{R}_{\#(S)}} \psi(S ; \mathbf{x}(S)) f_{\mathbf{C}(S)}^{\mathrm{No}}(\mathbf{x}(S)) \mathrm{d} \mathbf{x}(S), \quad \mathrm{A}(k)=\sum_{S \subseteq I^{(d)}: \#(S)=k} \alpha(S)
$$


and

$$
\lambda(S)=\alpha(S) \log \operatorname{det} \mathbf{C}(S), \Lambda(k):=\sum_{S \subseteq I^{(d)}: \#(S)=k} \lambda(S) .
$$

Consider the following condition invoking broken dependence and analogous to (2.8):

$$
\begin{aligned}
& \forall i \in S \subseteq I, \text { with } S_{i}^{-}=\{j \in S: j<i\} \text { and } S_{i}^{+}=\{j \in S: j>i\}, \\
& \quad \int_{\left(\mathbb{R}^{\#(S)}\right.} \psi(S ; \mathbf{x}(S))\left\{f_{\mathbf{C}(S)}^{\mathrm{No}}(\mathbf{x}(S))\right. \\
& \left.\quad-f_{\mathbf{C}\left(S_{i}^{-}\right)}^{\mathrm{No}}\left(\mathbf{x}\left(S_{i}^{-}\right)\right) \times\left[f_{\{i\} \mid S_{i}^{-}}^{\mathrm{No}}\left(x_{i} \mid \mathbf{x}\left(S_{i}^{-}\right)\right) f_{S_{i}^{+} \mid S_{i}^{-}}^{\mathrm{No}}\left(\mathbf{x}\left(S_{i}^{+}\right) \mid \mathbf{x}\left(S_{i}^{-}\right)\right)\right]\right\} \mathrm{d} \mathbf{x}(S) \geq 0,
\end{aligned}
$$

Theorem 5.5 (Cf. [2], Theorem 4 or [5], Theorem 31) Assume condition (5.12). Then the quantity $m(k)=m(k, \mathbf{C}, \phi)$ defined by

$$
m(k):=\left(\begin{array}{l}
d \\
k
\end{array}\right)^{-1}\left[\frac{\log \Lambda(k)}{k}+\frac{\log (2 \pi)}{2} A(k)+\frac{\log e}{2 k} \Lambda(k)\right]
$$

is decreasing in $k=1, \ldots, d$ :

$$
m(1) \geq \ldots \geq m(d)
$$

Proof. For $\mathbf{X}(S) \sim f_{\mathbf{C}(S)}^{\mathrm{No}}$ we have, by using (1.3):

$$
\frac{h_{\psi(S)}^{\mathrm{w}}(\mathbf{X}(S))}{k}=\frac{\alpha(S)}{2 k} \log \left[(2 \pi)^{k} \operatorname{det} \mathbf{C}(S)\right]+\frac{\log e}{2 k} \operatorname{tr}\left[\mathbf{C}(S)^{-1} \mathbf{\Phi}(S)\right] .
$$

Therefore,

$$
m(k)=\left(\begin{array}{l}
d \\
k
\end{array}\right)^{-1} \sum_{S:|S|=k}\left\{\frac{\alpha(S)}{2 k} \log \left[(2 \pi)^{k} \operatorname{det} \mathbf{C}(S)\right]+\frac{\log e}{2 k} \operatorname{tr}\left(\mathbf{C}(S)^{-1} \mathbf{\Phi}(S)\right)\right\}
$$

Invoking Theorem 2.2 completes the proof.

Theorem 5.6 (Cf. [2], Theorem 5 or [5], Theorem 32) Assuming (5.12), for all $r>0$ the values

$$
s(k)=s(k, \mathbf{C}, \phi):=\left(\begin{array}{l}
d \\
k
\end{array}\right)^{-1} \sum_{S \subseteq I^{(d)}: \#(S)=k} \Lambda(k)^{1 / k} \exp \left\{r\left[\frac{\log (2 \pi)}{2} A(k)+\frac{\log e}{2 k} \mathrm{~T}(k)\right]\right\}
$$

obey

$$
s(1) \geq \ldots \geq s(d)
$$


Proof. The assertion follows readily from Theorem 2.3.

Our next goal is to establish bounds for Toeplitz determinants extending Theorem 6 from [2] (or Theorem 27 from [5]). It is said that $\mathbf{C}=\left(C_{i j}\right)$ is a $d \times d$ Toeplitz matrix if $C_{i j}=C_{k l}$ whenever $|i-j|=|k-l|$. A more restrictive property is cyclic Toeplitz where $C_{i j}=C_{k l}$ whenever $\operatorname{dist}_{d}(i, j)=\operatorname{dist}_{d}(k, l)$. Here, for $1 \leq i<j \leq d$ the cyclic distance $\operatorname{dist}_{d}(i, j)=\min [j-i, d-j+i]$; it is then extended to a metric with $\operatorname{dist}_{d}(i, j)=\operatorname{dist}_{d}(j, i)$ and $\operatorname{dist}_{d}(i, i)=0$. As before, we consider sub-matrices $\mathbf{C}(S)$ where $S \subseteq I^{(d)}:=\{1, \ldots, d\}$ and the Gaussian random vectors $\mathbf{X}(S) \sim f_{\mathbf{C}(S)}^{\mathrm{No}}$ as sub-collections in $\mathbf{X}_{1}^{d}:=\left(\begin{array}{c}X_{1} \\ \vdots \\ X_{d}\end{array}\right) \sim f_{\mathbf{C}}^{\mathrm{No}}$. A special role is played by $S=I_{i, j}$ where $I_{i, j}$ stands for a segment of positive integers $\{i, i+1, \ldots, j\}$ of cardinality $j-i+1$ where $1 \leq i<j \leq d$. In particular, for $S=I_{1, k}$, we set: $\mathbf{C}(S)=\mathbf{C}_{k}$ and deal with vectors $\mathbf{X}_{1}^{k} \sim f_{\mathbf{C}_{k}}^{\text {No }}$, $1 \leq k \leq d$, with $\mathbf{C}_{d}=\mathbf{C}$.

Accordingly, we say that WF $\mathbf{x} \in \mathbb{R}^{d} \mapsto \phi(\mathbf{x}) \geq 0$ has a Toeplitz property if the value of the reduced WF $\psi\left(I_{i, j} ; \mathbf{x}_{i}^{j}\right)$ coincides with $\psi\left(I_{i+k, j+k} ; \mathbf{x}_{i+k}^{j+k}\right)$, provided that arguments $\mathbf{x}_{i}^{j}=\mathbf{x}\left(I_{i, j}\right)$ and $\mathbf{x}_{i+k}^{j+k}=\mathbf{x}\left(I_{i+k, j+k}\right)$ are shifts of each other, where $1 \leq i<j \leq d$ and $1 \leq i+k<j+k \leq d$. An example is where $\mathbf{C}$ is cyclic Toeplitz and $\phi$ has a product-form: $\phi(\mathbf{x})=\prod_{1 \leq i \leq d} \varphi\left(x_{i}\right)$. Recall, the reduced WF in question involves the conditional PDF $f_{I_{i, j}^{\mathrm{C}} \mid I_{i, j}}^{\mathrm{No}}\left(\mathbf{x}\left(I_{i, j}^{\complement}\right) \mid \mathbf{x}_{i}^{j}\right)$ :

$$
\psi\left(I_{i, j} ; \mathbf{x}_{i}^{j}\right)=\int_{\mathbb{R}^{d-j+i-1}} \phi(\mathbf{x}) f_{I_{i, j}^{\complement} \mid I_{i, j}}^{\mathrm{No}}\left(\mathbf{x}\left(I_{i, j}^{\complement}\right) \mid \mathbf{x}_{i}^{j}\right) \mathrm{d} \mathbf{x}\left(I_{i, j}^{\complement}\right) \text { where } I_{i, j}^{\complement}=I_{1, d} \backslash I_{i, j} .
$$

For $S=I_{1, k}, 1 \leq k \leq d$, in accordance with (1.3),

$$
h_{\psi(k)}\left(\mathbf{X}_{1}^{k}\right)=h_{\psi\left(I_{1, k}\right)}\left(\mathbf{X}_{1}^{k}\right)=\frac{\alpha\left(\mathbf{C}_{k}\right)}{2} \log \left[(2 \pi)^{k} \operatorname{det} \mathbf{C}_{k}\right]+\frac{\log e}{2} \operatorname{tr}\left[\mathbf{C}_{k}^{-1} \mathbf{\Psi}_{k}\right] .
$$

Here the value $\alpha\left(\mathbf{C}_{k}\right)=\alpha\left(\mathbf{C}_{k}, \mathbf{C}, \phi\right)$ and the $k \times k$ matrix $\boldsymbol{\Psi}_{k}=\boldsymbol{\Psi}_{k}\left(\mathbf{C}_{k}, \mathbf{C}, \psi\right)$ are given by

$$
\alpha\left(\mathbf{C}_{k}\right)=\int_{\mathbb{R}^{k}} \psi\left(k ; \mathbf{x}_{1}^{k}\right) f_{\mathbf{C}_{k}}^{\mathrm{No}}\left(\mathbf{x}_{1}^{k}\right) \mathrm{d} \mathbf{x}_{1}^{k}, \quad \mathbf{\Psi}_{k}=\int_{\mathbb{R}^{k}} \mathbf{x}_{1}^{k}\left(\mathbf{x}_{1}^{k}\right)^{\mathrm{T}} \psi\left(k ; \mathbf{x}_{1}^{k}\right) f_{\mathbf{C}_{k}}^{\mathrm{No}}\left(\mathbf{x}_{1}^{k}\right) \mathrm{d} \mathbf{x}_{1}^{k}
$$

and $\psi(k)=\psi\left(I_{1, k}\right)$. (For $k=d$, the subscript $k$ will be omitted.)

Theorem 5.7 (Cf. [2], Theorem 6 or [5], Theorem 27) Suppose $\mathbf{C}_{n}$ is a positive definite $d \times d$ Toeplitz matrix and $\phi$ has the Toeplitz property. Consider the map $k \in\{1, \ldots, d\} \mapsto a(k)=$ $a(k, \mathbf{C}, \phi)$ where

$$
a(k)=\alpha\left(C_{k}\right)\left\{\log (2 \pi)+\log \left[\left(\operatorname{det} \mathbf{C}_{k}\right)^{1 / k}\right]\right\}+\frac{\log e}{k} \operatorname{tr}\left[\mathbf{C}_{k}^{-1} \mathbf{\Psi}_{k}\right] .
$$

Assuming condition (5.12), the value $a(k)$ is decreasing in $k: a(1) \geq \ldots \geq a(d)$. 
Proof. By using the Toeplitz property of $\mathbf{C}$ and $\phi$, we can write

$$
h_{\psi\left(I_{1, k}\right)}^{\mathrm{w}}\left(X_{k} \mid \mathbf{X}_{1}^{k-1}\right)=h_{\psi\left(I_{2, k+1}\right)}^{\mathrm{w}}\left(X_{k+1} \mid \mathbf{X}_{2}^{k}\right) .
$$

Next, Theorem 4.3 yields:

$$
h_{\psi\left(I_{2, k+1}\right)}^{\mathrm{w}}\left(X_{k+1} \mid \mathbf{X}_{2}^{k}\right) \geq h_{\psi\left(I_{1, k+1}\right)}^{\mathrm{w}}\left(X_{k+1} \mid \mathbf{X}_{1}^{k}\right) .
$$

From (5.18) and (5.19) we conclude that $h_{\psi\left(I_{1, k}\right)}^{\mathrm{w}}\left(X_{k} \mid \mathbf{X}_{1}^{k-1}\right)$ is decreasing in $k$. Thus the running average also decreases. On the other hand, by the chain rule

$$
\frac{1}{k} h_{\psi\left(I_{1, k}\right)}\left(\mathbf{X}_{1}^{k}\right)=\frac{1}{k} \sum_{i=1}^{k} h_{\psi\left(I_{1, i}\right)}^{\mathrm{w}}\left(X_{i} \mid \mathbf{X}_{1}^{i-1}\right) .
$$

Consequently $\frac{1}{k} h_{\psi\left(I_{1, k}\right)}\left(\mathbf{X}_{1}^{k}\right)$ too decreases in $k$. Referring to Eqns (5.16) and (55.15) leads directly to the result.

Theorem 5.8 (Cf. [5], Theorem 33.) Given a WF $\mathbf{x}=\left(\begin{array}{c}x_{1} \\ \vdots \\ x_{d}\end{array}\right) \in \mathbb{R}^{d} \mapsto \phi(\mathbf{x})$, assume condition

$$
\int_{\mathbb{R}^{d}} \phi(\mathbf{x})\left[f_{C}^{\mathrm{No}}(\mathbf{x})-\prod_{i=1}^{n} f_{C_{i i}}^{\mathrm{No}}\left(x_{i}\right)\right] \mathrm{d} \mathbf{x} \geq 0 .
$$

Then the quantity

$$
\begin{aligned}
w(k) & =w(k, \mathbf{C}, \phi)=\left(\begin{array}{l}
d \\
k
\end{array}\right)^{-1} \frac{\alpha(\mathbf{C})}{2 k} \log \left[\prod_{S \subseteq I_{n}: \#(S)=k} \frac{(2 \pi)^{d}(\operatorname{det} \mathbf{C})}{(2 \pi)^{d-k}\left(\operatorname{det} \mathbf{C}\left(S^{\complement}\right)\right)}\right] \\
& +\left(\begin{array}{l}
d \\
k
\end{array}\right)^{-1} \frac{\log e}{2 k} \sum_{S \subseteq I_{n}: \#(S)=k}\left\{\operatorname{tr}\left[\mathbf{C}^{-1} \mathbf{\Phi}\right]-\operatorname{tr}\left[\mathbf{C}\left(S^{\complement}\right)^{-1} \mathbf{\Phi}\left(S^{\complement}\right)\right]\right\}
\end{aligned}
$$

is increasing in $k$, with

$$
w(1) \leq \cdots \leq w(d) .
$$

Proof. Using the conditional WE, we can write

$$
\begin{aligned}
h_{\phi}^{\mathrm{w}}\left(\mathbf{X}(S) \mid \mathbf{X}\left(S^{\complement}\right)\right) & =h_{\phi}^{\mathrm{w}}\left(\mathbf{X}(S), \mathbf{X}\left(S^{\complement}\right)\right)-h_{\psi\left(S^{\complement}\right)}^{\mathrm{w}}\left(\mathbf{X}\left(S^{\complement}\right)\right) \\
& =\frac{\alpha(\mathbf{C})}{2} \log \left[(2 \pi)^{d}(\operatorname{det} \mathbf{C})\right]+\frac{\log e}{2} \operatorname{tr}\left[\mathbf{C}^{-1} \mathbf{\Phi}\right] \\
& -\frac{\alpha(\mathbf{C})}{2} \log \left[(2 \pi)^{d-k}\left(\operatorname{det} \mathbf{C}\left(S^{\complement}\right)\right)\right]+\frac{\log e}{2} \operatorname{tr}\left[\mathbf{C}\left(S^{\complement}\right)^{-1} \mathbf{\Phi}\left(S^{\complement}\right)\right] .
\end{aligned}
$$


Here $\alpha(\mathbf{C})=\int_{\mathbb{R}^{d}} \phi(\mathbf{x}) f_{\mathbf{C}}^{\mathrm{No}}(\mathbf{x}) \mathrm{d} \mathbf{x}=\int_{\mathbb{R}^{\#\left(S^{\complement}\right)}} \psi\left(\mathbf{x}\left(S^{\complement}\right)\right) f_{\mathbf{C}\left(S^{\complement}\right)}^{\mathrm{No}}\left(\mathbf{x}\left(S^{\complement}\right)\right) \mathrm{d} \mathbf{x}\left(S^{\complement}\right)$. Therefore,

$$
\begin{aligned}
& h_{\phi}^{\mathrm{w}}\left(\mathbf{X}(S) \mid \mathbf{X}\left(S^{\complement}\right)\right) \\
& \quad=\frac{\alpha(\mathbf{C})}{2} \log \left[\frac{(2 \pi)^{d}(\operatorname{det} \mathbf{C})}{(2 \pi)^{d-k}\left(\operatorname{det} \mathbf{C}\left(S^{\complement}\right)\right)}\right]+\frac{\log e}{2}\left\{\operatorname{tr}\left[\mathbf{C}^{-1} \mathbf{\Phi}\right]-\operatorname{tr}\left[\mathbf{C}\left(S^{\complement}\right)^{-1} \mathbf{\Phi}\left(S^{\complement}\right)\right]\right\} .
\end{aligned}
$$

After that we apply Theorem 2.4 which completes the proof.

Remark 5.9 Note that the outermost inequality, $w(1) \leq w(d)$, can be rewritten as

$$
\begin{array}{r}
\alpha(\mathbf{C}) \log \left[(2 \pi)^{d}(\operatorname{det} \mathbf{C})\right]+\log e \operatorname{tr}\left[\mathbf{C}^{-1} \mathbf{\Phi}\right] \geq \alpha(\mathbf{C}) \log \left[\prod_{i=1}^{d} \frac{2 \pi(\operatorname{det} \mathbf{C})}{\operatorname{det} \mathbf{C}\left(I_{1}^{i-1} \cup I_{i+1}^{d}\right)}\right] \\
+\log e \sum_{i=1}^{d}\left\{\operatorname{tr}\left[\mathbf{C}^{-1} \mathbf{\Phi}\right]-\operatorname{tr}\left[\mathbf{C}\left(I_{1}^{i-1} \cup I_{i+1}^{d}\right)^{-1} \mathbf{\Phi}\left(I_{1}^{i-1} \cup I_{i+1}^{d}\right)\right]\right\} .
\end{array}
$$

Our next goal is to establish additional WDIs by using Theorem 2.6. For this purpose, we first analyse the mutual Gaussian WE, $i_{\phi}^{\mathrm{w}}\left(\mathbf{X}(S): \mathbf{X}\left(S^{\complement}\right)\right)$. According to the definition of the mutual WE in [9], we can write

$$
i_{\phi}^{\mathrm{w}}\left(\mathbf{X}(S): \mathbf{X}\left(S^{\complement}\right)\right)=h_{\psi(S)}^{\mathrm{w}}(\mathbf{X}(S))-h_{\phi}^{\mathrm{w}}\left(\mathbf{X}(S) \mid \mathbf{X}\left(S^{\complement}\right)\right) .
$$

Then, in accordance with (5.22), we have

$$
\begin{aligned}
& i_{\phi}^{\mathrm{w}}\left(\mathbf{X}(S): \mathbf{X}\left(S^{\complement}\right)\right)=\frac{\alpha(\mathbf{C})}{2} \log \left[\frac{(\operatorname{det} \mathbf{C}(S))\left(\operatorname{det} \mathbf{C}\left(S^{\complement}\right)\right)}{(\operatorname{det} \mathbf{C})}\right] \\
& \quad+\frac{\log e}{2}\left\{\operatorname{tr}\left[\mathbf{C}(S)^{-1} \mathbf{\Phi}(S)\right]+\operatorname{tr}\left[\mathbf{C}\left(S^{\complement}\right)^{-1} \mathbf{\Phi}\left(S^{\complement}\right)\right]-\operatorname{tr}\left[\mathbf{C}^{-1} \mathbf{\Phi}\right]\right\} .
\end{aligned}
$$

In Theorems 5.10 and 5.11 we consider the following condition (5.24) stemming from (2.20): $\forall S \subseteq\{1, \ldots, n\}$ with $\# S \geq 2$ and $i, j \in S$ with $i \neq j$,

$$
\begin{aligned}
& \int_{\mathbb{R}^{d}} \phi(\mathbf{x}) f_{S^{\complement} \mid S}^{\mathrm{No}}\left(\mathbf{x}\left(S^{\complement}\right) \mid \mathbf{x}(S)\right)\left[f_{\mathbf{C}(S)}^{\mathrm{No}}(\mathbf{x}(S))\right. \\
& \quad-f_{\mathbf{C}(S \backslash\{i, j\})}^{\mathrm{No}}\left(\mathbf { x } ( S \backslash \{ i , j \} ) f _ { i | S \backslash \{ i , j \} } ^ { \mathrm { No } } \left(\mathbf{x}(S \backslash\{i, j\}) f_{j \mid S \backslash\{i, j\}}^{\mathrm{No}}\left(x_{j} \mid \mathbf{x}(S \backslash\{i, j\})\right] \mathrm{d} \mathbf{x} \geq 0 .\right.\right.
\end{aligned}
$$

The proof of Theorems 5.10 and 5.11 is done with the help of Theorem 2.6, assuming that $X_{1}, X_{2}, \ldots, X_{d}$ are normally distributed with covariance matrix $\mathbf{C}$.

Theorem 5.10 (Cf. [5], Theorem 34.) Assume condition (5.24). Let

$$
\begin{aligned}
u(k) & =\left(\begin{array}{l}
d \\
k
\end{array}\right)^{-1} \frac{\alpha(\mathbf{C})}{2 k} \log \left[\prod_{S \subseteq I^{(d)}: \#(S)=k} \frac{(\operatorname{det} \mathbf{C}(S))\left(\operatorname{det} \mathbf{C}\left(S^{\complement}\right)\right)}{(\operatorname{det} \mathbf{C})}\right] \\
& +\left(\begin{array}{l}
d \\
k
\end{array}\right)^{-1} \frac{\log e}{2 k} \sum_{S \subseteq I_{n}: \#(S)=k}\left\{\operatorname{tr}\left[\mathbf{C}(S)^{-1} \mathbf{\Phi}(S)\right]+\operatorname{tr}\left[\mathbf{C}\left(S^{\complement}\right)^{-1} \mathbf{\Phi}\left(S^{\complement}\right)\right]-\operatorname{tr}\left[\mathbf{C}^{-1} \boldsymbol{\Phi}\right]\right\} .
\end{aligned}
$$


Then

$$
u(1) \geq u(2) \geq \cdots \geq u(d-1) \geq u(d) .
$$

Theorem 5.11 (Cf. [5], Theorem 35.) Under condition (5.24), let

$$
\begin{aligned}
z(k) & =\left(\begin{array}{l}
d \\
k
\end{array}\right)^{-1} \frac{\alpha(\mathbf{C})}{2} \log \left[\prod_{S \subseteq I^{(d)}: \#(S)=k} \frac{(\operatorname{det} \mathbf{C}(S))\left(\operatorname{det} \mathbf{C}\left(S^{\complement}\right)\right)}{(\operatorname{det} \mathbf{C})}\right] \\
& +\left(\begin{array}{l}
d \\
k
\end{array}\right)^{-1} \frac{\log e}{2} \sum_{S \subseteq I^{(d)}: \#(S)=k}\left\{\operatorname{tr}\left[\mathbf{C}(S)^{-1} \mathbf{\Phi}(S)\right]+\operatorname{tr}\left[\mathbf{C}\left(S^{\complement}\right)^{-1} \mathbf{\Phi}\left(S^{\complement}\right)\right]-\operatorname{tr}\left[\mathbf{C}^{-1} \mathbf{\Phi}\right]\right\} .
\end{aligned}
$$

Then

$$
z(1) \geq z(2) \geq \cdots \geq z(\lfloor d / 2\rfloor) .
$$

\section{Weighted Hadamard-type inequalities}

In this section we group several results related to the weighted Hadamard inequality (WHI); cf. [9], Theorem 3.3. The WHI inequality asserts that for a $d \times d$ positive definite matrix $\mathbf{C}$, under condition (5.20) we have:

$$
\alpha(\mathbf{C}) \log \prod_{i} \mathbf{C}_{i i}+(\log e) \sum_{i} \mathbf{C}_{i i}^{-1} \Phi_{i i}-\alpha(\mathbf{C}) \log \operatorname{det} \mathbf{C}-(\log e) \operatorname{tr} \mathbf{C}^{-1} \mathbf{\Phi} \geq 0,
$$

with equality iff $\mathbf{C}$ is diagonal. Recall, $\alpha(\mathbf{C})=\alpha_{\phi}(\mathbf{C})$ and $\mathbf{\Phi}=\mathbf{\Phi}_{\mathbf{C}}=\Phi_{\mathbf{C}, \phi}$ are as in (1.4).

We begin with the weighted version of the strong Hadamard inequality (WSHI). The inequality (and other bounds in this section) will involve determinants $\operatorname{det} \mathbf{C}(S)$ of sub-matrices $\mathbf{C}(S)$ in $\mathbf{C}$ where, as before, $S$ is a subset of $I^{(d)}:=\{1, \ldots, d\}$ of a special type. Namely, we fix $p \in\{1, \ldots, d-1\}$ and consider the segment $I_{p+1, d}=\{p+1, \ldots, d\}$, segment $I_{1, p}=\{1, \ldots, p\}$ and unions $\{i\} \cup I_{p+1, d}$ and $I_{1, i} \cup I_{p+1, d}=I_{i+1, p}^{\complement}$ where $i \in I_{1, p}$. We deal with the related entry $C_{i i}$ in $\mathbf{C}$ and sub-matrices

$$
\mathbf{C}_{p+1}^{d}:=\mathbf{C}\left(I_{p+1, d}\right), \mathbf{C}_{1}^{i-1}:=\mathbf{C}\left(I_{1, i-1}\right), \mathbf{C}\left(\{i\} \cup I_{p+1, d}\right) \text { and } \mathbf{C}\left(I_{1, i} \cup I_{p+1, d}\right)
$$

and Gaussian random variables $X_{i}$ and vectors $\mathbf{X}_{p+1}^{d}:=\mathbf{X}\left(I_{p+1, d}\right), \mathbf{X}_{1}^{i-1}:=\mathbf{X}\left(I_{1, i-1}\right)$, $X_{i} \vee \mathbf{X}_{p+1}^{d}:=\mathbf{X}\left(\{i\} \cup I_{p+1, d}\right)$ and $\mathbf{X}_{1}^{i} \vee \mathbf{X}_{p+1}^{d}:=\mathbf{X}\left(I_{1, i} \cup I_{p+1, d}\right)$ using symbols $x_{i}, \mathbf{x}_{p+1}^{d}, \mathbf{x}_{1}^{i-1}$, and $\mathbf{x}_{1}^{i} \vee \mathbf{x}_{p+1}^{d}$ for their respective values. Thus, PDFs

$$
f_{\mathbf{X}_{p+1}^{d}}\left(\mathbf{x}_{p+1}^{d}\right)=f_{\mathbf{C}_{p+1}^{d}}^{\text {No }}\left(\mathbf{x}_{p+1}^{d}\right) \text { and } f_{\mathbf{X}_{1}^{i} \vee \mathbf{X}_{p+1}^{d}}\left(\mathbf{x}_{1}^{i} \vee \mathbf{x}_{p+1}^{d}\right)=f_{\mathbf{C}\left(I_{1, i-1} \cup I_{p+1, d}\right)}^{\mathrm{No}}\left(\mathbf{x}_{1}^{i} \vee \mathbf{x}_{p+1}^{d}\right)
$$

emerge, as well as conditional PDFs $f_{X_{i} \mid \mathbf{X}_{p+1}^{d}}\left(x_{i} \mid \mathbf{x}_{p+1}^{d}\right)$ and $f_{\mathbf{X}_{1}^{i-1} \mid \mathbf{X}_{p+1}^{d}}\left(\mathbf{x}_{1}^{i-1} \mid \mathbf{x}_{p+1}^{d}\right)$. Viz., $\mathbf{X}_{1}^{i} \vee$ $\mathbf{X}_{p+1}^{d}$ and $\mathbf{x}_{1}^{i} \vee \mathbf{x}_{p+1}^{d}$ stand for the concatenated vectors $\left(\begin{array}{c}X_{1} \\ \vdots \\ X_{i} \\ X_{p+1} \\ \vdots \\ X_{d}\end{array}\right)$ and $\left(\begin{array}{c}x_{1} \\ \vdots \\ x_{i} \\ x_{p+1} \\ \vdots \\ x_{d}\end{array}\right)$, each with 
$i+d-p$ entries. As above (see (1.4)), for a given WF $\mathbf{x} \in \mathbb{R}^{d} \mapsto \phi(\mathbf{x})$ we consider numbers $\alpha\left(\mathbf{C}_{1}^{p}\right)=\alpha_{\phi}\left(\mathbf{C}_{1}^{p}\right)$ and matrices $\boldsymbol{\Phi}_{\mathbf{C}_{1}^{p}}=\mathbf{\Phi}_{\mathbf{C}_{1}^{p}, \mathbf{C}, \phi}$ :

$$
\alpha\left(\mathbf{C}_{1}^{p}\right)=\alpha_{\phi}\left(\mathbf{C}_{1}^{p}\right)=\int_{\mathbb{R}^{d}} \phi\left(\mathbf{x}_{1}^{d}\right) f_{\mathbf{C}_{1}^{p}}^{\mathrm{No}}\left(\mathbf{x}_{1}^{p}\right) \mathrm{d} \mathbf{x}, \quad \mathbf{\Phi}_{\mathbf{C}_{1}^{p}}=\mathbf{\Phi}_{\mathbf{C}_{1}^{p}, \mathbf{C}, \phi}=\int_{\mathbb{R}^{d}} \mathbf{x}_{1}^{p}\left(\mathbf{x}_{1}^{p}\right)^{\mathrm{T}} \phi(\mathbf{x}) f_{\mathbf{C}}^{\mathrm{No}}(\mathbf{x}) \mathrm{d} \mathbf{x} .
$$

(In Eqns (6.16) and (6.22) - (6.24) we will use variations of these formulas.) We also set

$$
\begin{aligned}
& \boldsymbol{\Phi}_{p+1}^{d}=\int_{\mathbb{R}^{p-d}} \mathbf{x}_{p+1}^{d}\left(\mathbf{x}_{p+1}^{d}\right)^{\mathrm{T}} \psi\left(I_{p+1, d} ; \mathbf{x}_{p+1}^{d}\right) f_{\mathbf{X}_{p+1}^{d}}\left(\mathbf{x}_{p+1}^{d}\right) \mathrm{d} \mathbf{x}_{p+1}^{d}, \\
& \boldsymbol{\Phi}\left(\{i\} \cup I_{p+1, d}\right)=\int_{\mathbb{R}^{p-d+1}}\left(x_{i} \vee \mathbf{x}_{p+1}^{d}\right)\left(x_{i} \vee \mathbf{x}_{p+1}^{d}\right)^{\mathrm{T}} \\
& \quad \times \psi\left(\{i\} \cup I_{p+1, d} ; x_{i} \vee \mathbf{x}_{p+1}^{d}\right) f_{X_{i} \vee \mathbf{X}_{p+1}^{d}}\left(x_{i} \vee \mathbf{x}_{p+1}^{d}\right) \mathrm{d}\left(x_{i} \vee \mathbf{x}_{p+1}^{d}\right),
\end{aligned}
$$

with reduced WFs $\psi\left(I_{p+1, d}\right)$ and $\psi\left(\{i\} \cup I_{p+1, d}\right)$ calculated as in (2.5) , for $S=I_{p+1, d}$ and $S=\{i\} \cup I_{p+1, d}$.

Furthermore, we will assume in Theorem 6.1 that, $\forall i=1, \ldots, p$, the reduced WF $\psi(S)$ with $S=\{1, \ldots i, p+1, \ldots d\}=I_{i+1, p}^{\complement}$ obeys

$$
\begin{aligned}
& \int_{\mathbb{R}^{i+d-p}} \psi\left(I_{i+1, p}^{\complement} ; \mathbf{x}_{1}^{i} \vee \mathbf{x}_{p+1}^{d}\right)\left\{f_{\mathbf{X}_{1}^{i} \vee \mathbf{X}_{p+1}^{d}}\left(\mathbf{x}_{1}^{i} \vee \mathbf{x}_{p+1}^{d}\right)\right. \\
& \left.\quad-f_{\mathbf{X}_{p+1}^{d}}\left(\mathbf{x}_{p+1}^{d}\right) \times\left[f_{X_{i} \mid \mathbf{X}_{p+1}^{d}}^{\mathrm{No}}\left(x_{i} \mid \mathbf{x}_{p+1}^{d}\right) f_{\mathbf{X}_{1}^{i-1} \mid \mathbf{X}_{p+1}^{d}}\left(\mathbf{x}_{1}^{i-1} \mid \mathbf{x}_{p+1}^{d}\right)\right]\right\} \mathrm{d}\left(\mathbf{x}_{1}^{i} \vee \mathbf{x}_{p+1}^{d}\right) \geq 0 .
\end{aligned}
$$

The 'standard' SHI is

$$
\begin{aligned}
& \frac{\operatorname{det} \mathbf{C}}{\operatorname{det} \mathbf{C}_{p+1}^{d}} \leq \prod_{1 \leq i \leq p} \frac{\operatorname{det} \mathbf{C}\left(\{i\} \cup I_{p+1, d}\right)}{\operatorname{det} \mathbf{C}_{p+1}^{d}} \\
& \quad \text { or } \log \operatorname{det} \mathbf{C}+(p-1) \log \operatorname{det} \mathbf{C}_{p+1}^{d} \leq \sum_{1 \leq i \leq p} \log \operatorname{det} \mathbf{C}\left(\{i\} \cup I_{p+1, d}\right) .
\end{aligned}
$$

The WE approach offers the following WSHI:

Theorem 6.1 (Cf. [2], Theorem 8 or [5], Theorem 28.) Under condition (6.3), for $1 \leq p<d$,

$$
\begin{gathered}
\alpha(\mathbf{C}) \log \left[(2 \pi)^{d} \operatorname{det} \mathbf{C}\right]+(\log e) \operatorname{tr}\left(\mathbf{C}^{-1} \mathbf{\Phi}\right) \\
+(p-1)\left\{\alpha\left(\mathbf{C}_{p+1}^{d}\right) \log \left[(2 \pi)^{d-p} \operatorname{det} \mathbf{C}_{p+1}^{d}\right]+(\log e) \operatorname{tr}\left[\left(\mathbf{C}_{p+1}^{d}\right)^{-1} \mathbf{\Phi}_{p+1}^{d}\right]\right\} \\
\leq \sum_{1 \leq i \leq p}\left\{\alpha\left(\mathbf{C}\left(\{i\} \cup I_{p+1, d}\right)\right) \log \left[(2 \pi)^{d-p+1} \operatorname{det} \mathbf{C}\left(\{i\} \cup I_{p+1, d}\right)\right]\right. \\
\left.+(\log e) \operatorname{tr}\left[\mathbf{C}\left(\{i\} \cup I_{p+1, d}\right)^{-1} \mathbf{\Phi}\left(\{i\} \cup I_{p+1, d}\right)\right]\right\} .
\end{gathered}
$$

Proof. We use the same idea as in Theorem 3.3 from [9]. Recalling ([6.9) we can write

$$
\begin{aligned}
h_{\phi}^{\mathrm{w}}\left(\mathbf{X}_{1}^{p} \mid \mathbf{X}_{p+1}^{d}\right) & =\frac{1}{2} \log \left[(2 \pi)^{d} \operatorname{det} \mathbf{C}\right] \alpha(\mathbf{C})+\frac{\log e}{2} \operatorname{tr}\left(\mathbf{C}^{-1} \mathbf{\Phi}\right) \\
& -\frac{1}{2} \log \left[(2 \pi)^{d-p} \operatorname{det} \mathbf{C}_{p+1}^{d}\right] \alpha\left(\mathbf{C}_{p+1}^{d}\right)-\frac{\log e}{2} \operatorname{tr}\left[\left(\mathbf{C}_{p+1}^{d}\right)^{-1} \mathbf{\Phi}_{p+1}^{d}\right],
\end{aligned}
$$


Cf. Eqns (5.9), (5.10), (5.16). Furthermore, by subadditivity of the conditional WE (see 99], Theorem 1.4), under assumption (6.3) we can write

$$
h_{\phi}^{\mathrm{w}}\left(\mathbf{X}_{1}^{p} \mid \mathbf{X}_{p+1}^{d}\right) \leq \sum_{i=1}^{p} h_{\psi\left(\{i\} \cup I_{p+1, d}\right)}^{\mathrm{w}}\left(X_{i} \mid \mathbf{X}_{p+1}^{d}\right) .
$$

Here for $i=1, \ldots, p$, again in agreement with (6.9),

$$
\begin{gathered}
h_{\psi\left(\{i\} \cup I_{p+1, d}\right)}^{\mathrm{w}}\left(X_{i} \mid \mathbf{X}_{p+1}^{d}\right)=\frac{1}{2} \log \left[(2 \pi)^{d-p+1} \operatorname{det} \mathbf{C}\left(\{i\} \cup I_{p+1, d}\right)\right] \alpha\left(\mathbf{C}\left(\{i\} \cup I_{p+1, d}\right)\right) \\
+\frac{\log e}{2} \operatorname{tr} \mathbf{C}\left(\{i\} \cup I_{p+1, d}\right)^{-1} \mathbf{\Phi}\left(\{i\} \cup I_{p+1, d}\right) \\
-\frac{1}{2} \log \left[(2 \pi)^{d-p} \operatorname{det} \mathbf{C}_{p+1}^{d}\right] \alpha\left(\mathbf{C}_{p+1}^{d}\right)-\frac{\log e}{2} \operatorname{tr}\left[\left(\mathbf{C}_{p+1}^{d}\right)^{-1} \boldsymbol{\Phi}_{p+1}^{d}\right] .
\end{gathered}
$$

Substituting into (6.6) yields the assertion of the theorem.

Our next result, Theorem 6.2, gives an extension of Lemma 9 from [2] (or Lemma 8 from [5]). The latter asserts that an individual diagonal entry $C_{i i}$ of a $d \times d$ positive definite matrix equals the ratio of the relevant determinants, viz.,

$$
C_{d d}=\frac{\operatorname{det} \mathbf{C}}{\operatorname{det} \mathbf{C}_{1}^{d-1}}, \quad \text { or } \log C_{d d}+\log \operatorname{det} \mathbf{C}_{1}^{d-1}-\log \operatorname{det} \mathbf{C}=0 .
$$

Remarkably, Theorem 6.2 does not require assumption 6.3).

Theorem 6.2 (Cf. [2], Lemma 9 or [5], Lemma 8.) The following equality holds true:

$$
\begin{array}{r}
\alpha\left(C_{d d}\right) \log \left[(2 \pi) C_{d d}\right]+\alpha\left(\mathbf{C}_{1}^{d-1}\right) \log \left[(2 \pi)^{d-1} \operatorname{det} \mathbf{C}_{1}^{d-1}\right]-\alpha(\mathbf{C}) \log \left[(2 \pi)^{d} \operatorname{det} \mathbf{C}\right] \\
=(\log e) \operatorname{tr}\left[\mathbf{C}^{-1} \mathbf{\Phi}\right]-(\log e) \operatorname{tr}\left[\left(\mathbf{C}_{1}^{d-1}\right)^{-1} \mathbf{\Phi}_{1}^{d-1}\right]-(\log e) C_{d d}^{-1} \Phi_{d d}
\end{array}
$$

Proof. Using the conditional normality of $X_{d}$ given $\mathbf{X}_{1}^{d-1}$, we can write

$$
h_{\phi}^{\mathrm{w}}\left(X_{d} \mid \mathbf{X}_{1}^{d-1}\right)=\frac{\alpha\left(C_{d d}\right)}{2} \log \left[(2 \pi) C_{d d}^{2}\right]+\frac{\log e}{2} C_{d d}^{2} \Phi_{d d} .
$$

On the other hand,

$$
h_{\phi}^{\mathrm{w}}\left(X_{d} \mid \mathbf{X}_{1}^{d-1}\right)=h_{\phi}^{\mathrm{w}}\left(\mathbf{X}_{1}^{d}\right)-h_{\psi\left(I_{1, d-1}\right)}^{\mathrm{w}}\left(\mathbf{X}_{1}^{d-1}\right)
$$

and therefore

$$
\begin{aligned}
& \frac{\alpha\left(C_{d d}\right)}{2} \log \left[(2 \pi) C_{d d}^{2}\right]+\frac{\log e}{2} C_{d d}^{2} \Phi_{d d} \\
& \quad=\frac{\alpha(\mathbf{C})}{2} \log \left[(2 \pi)^{d} \operatorname{det} \mathbf{C}\right]+\frac{\log e}{2} \operatorname{tr} \mathbf{C} \mathbf{\Phi} \\
& \quad-\frac{\alpha\left(\mathbf{C}_{1}^{d-1}\right)}{2} \log \left[(2 \pi)^{d-1} \operatorname{det} \mathbf{C}_{1}^{d-1}\right]-\frac{\log e}{2} \operatorname{tr}\left[\left(\mathbf{C}_{1}^{d-1}\right)^{-1} \mathbf{\Phi}_{1}^{(d-1)}\right] .
\end{aligned}
$$

The result then follows. 
The next assertion, Theorem 6.3, extends the result of Theorem 9 from [2] (or Theorem 29 from [5]) that, $\forall p=1, \ldots, d, \mathbf{C} \mapsto \log \frac{\operatorname{det} \mathbf{C}}{\operatorname{det} \mathbf{C}_{1}^{p}}$ is a concave function of a positive definite $d \times d$ matrix C. We will write matrix $\mathbf{C}$ in the block form similar to (3.3):

$$
\mathbf{C}=\left(\begin{array}{cc}
\mathbf{C}_{1}^{p} & \mathbf{C}_{n-p}^{p} \\
\mathbf{C}_{p}^{n-p} & \mathbf{C}_{p+1}^{d}
\end{array}\right)
$$

Set $\mathbf{D} \mathbf{x}_{p+1}^{d}=\mathbf{C}_{p}^{d-p}\left(\mathbf{C}_{p+1}^{d}\right)^{-1} \mathbf{x}_{p+1}^{d}$ and $\mathbf{B}_{1}^{p}=\mathbf{C}_{1}^{p}-\mathbf{C}_{p}^{d-p}\left(\mathbf{C}_{p+1}^{d}\right)^{-1} \mathbf{C}_{d-p}^{p}$. Consider the following inequalities

$$
\int_{\mathbb{R}^{d}} \phi(\mathbf{x}) f_{\mathbf{X}_{1}^{p}}\left(\mathbf{x}_{1}^{p}\right)\left[f_{\mathbf{X}_{p+1}^{d} \mid \mathbf{X}_{1}^{p}}\left(\mathbf{x}_{p+1}^{d} \mid \mathbf{x}_{1}^{p}\right)-f_{\mathbf{Y}_{p+1}^{d}}^{\mathrm{No}} \mid \mathbf{Y}_{1}^{p}\left(\mathbf{x}_{p+1}^{d} \mid \mathbf{x}_{1}^{p}\right)\right] \mathrm{d} \mathbf{x} \geq 0
$$

and

$$
\begin{aligned}
\int_{\mathbb{R}^{d}} \phi(\mathbf{x})[ & \left.f_{\mathbf{X}}(\mathbf{x})-f_{\mathbf{C}}^{\mathrm{No}}(\mathbf{x})\right]\left\{\log \left[(2 \pi)^{p} \operatorname{det}\left(\mathbf{B}_{1}^{p}\right)^{-1}\right]\right. \\
& \left.+(\log e)\left[\left(\mathbf{x}_{1}^{p}-\mathbf{D} \mathbf{x}_{p+1}^{d}\right)^{\mathrm{T}}\left(\mathbf{B}_{1}^{p}\right)^{-1}\left(\mathbf{x}_{1}^{p}-\mathbf{D} \mathbf{x}_{p+1}^{d}\right)\right]\right\} \mathrm{d} \mathbf{x} \leq 0 .
\end{aligned}
$$

Theorem 6.3 (Cf. [2], Theorem 9 or [5], Theorem 29.) Assume that $\mathbf{C}=\lambda \mathbf{C}^{\prime}+(1-\lambda) \mathbf{C}^{\prime \prime}$ where $\mathbf{C}, \mathbf{C}^{\prime}$ and $\mathbf{C}^{\prime \prime}$ are positive definite $d \times d$ matrices and $\lambda \in[0,1]$. Given a WF $\mathbf{x} \mapsto \phi(\mathbf{x}) \geq 0$ and $1 \leq p \leq d$, define:

$$
\begin{aligned}
\mu(\mathbf{C})= & \alpha(\mathbf{C}) \log \left[(2 \pi)^{d} \operatorname{det} \mathbf{C}\right]+(\log e) \operatorname{tr}\left[\mathbf{C}^{-1} \boldsymbol{\Phi}_{\mathbf{C}}\right] \\
& -\alpha\left(\mathbf{C}_{1}^{p}\right) \log \left[(2 \pi)^{d} \operatorname{det} \mathbf{C}_{1}^{p}\right]-(\log e) \operatorname{tr}\left[\left(\mathbf{C}_{1}^{p}\right)^{-1} \mathbf{\Phi}_{\mathbf{C}_{1}^{p}}\right]
\end{aligned}
$$

and similarly with $\mu\left(\mathbf{C}^{\prime}\right)$ and $\mu\left(\mathbf{C}^{\prime \prime}\right)$. Then

$$
\mu(\mathbf{C}) \geq \lambda \mu\left(\mathbf{C}^{\prime}\right)+(1-\lambda) \mu\left(\mathbf{C}^{\prime \prime}\right) .
$$

Proof. Again we essentially follow the method from 2] with modifications developed in 9]. Fix two $d \times d$ positive definite matrices $\mathbf{C}^{\prime}$ and $\mathbf{C}^{\prime \prime}$ and set $\mathbf{X}^{\prime} \sim f_{\mathbf{C}^{\prime}}^{\mathrm{No}}, \mathbf{X}^{\prime \prime} \sim f_{\mathbf{C}^{\prime \prime}}^{\text {No. Given }}$ $\lambda \in[0,1]$, consider a random variable $\Theta$ taking values $\vartheta=1,2$ with probabilities $\lambda$ and $1-\lambda$ independently of $\left(\mathbf{X}^{\prime}, \mathbf{X}^{\prime \prime}\right)$. Next, set

$$
\mathbf{X}= \begin{cases}\mathbf{X}^{\prime}, & \text { when } \Theta=1 \\ \mathbf{X}^{\prime \prime}, & \text { when } \Theta=2 .\end{cases}
$$

Then $\mathbf{X} \sim\left(\lambda f_{\mathbf{C}^{\prime}}^{\mathrm{No}}+(1-\lambda) f_{\mathbf{C}^{\prime \prime}}^{\mathrm{No}}\right)$ and the covariance matrix $\operatorname{Cov} \mathbf{X}=\lambda \mathbf{C}^{\prime}+(1-\lambda) \mathbf{C}^{\prime \prime}=: \mathbf{C}$.

With the WF $\widetilde{\phi}\left(\mathbf{x}_{1}^{d}, \vartheta\right)=\phi\left(\mathbf{z}_{1}^{d}\right)$, use Theorem 2.1 from [9] and Theorem 3.2 from Section 3 and write:

$$
h_{\widetilde{\phi}}^{\mathrm{w}}\left(\mathbf{X}_{p+1}^{d} \mid \mathbf{X}_{1}^{p}, \Theta\right) \leq h_{\phi}^{\mathrm{w}}\left(\mathbf{X}_{p+1}^{d} \mid \mathbf{X}_{1}^{p}\right) \leq h_{\phi}^{\mathrm{w}}\left(\mathbf{Y}_{p+1}^{d} \mid \mathbf{Y}_{1}^{p}\right)
$$


Here $\mathbf{Y}$ stands for the Gaussian random vector with the $\operatorname{PDF} f_{\mathbf{C}}^{\mathrm{No}}\left(\mathbf{x}_{1}^{d}\right)$. The LHS in (6.15) coincides with $\lambda \mu\left(\mathbf{C}^{\prime}\right)+(1-\lambda) \mu\left(\mathbf{C}^{\prime \prime}\right)$ and the RHS with $\mu(\mathbf{C})$. This completes the proof.

In a particular case $p=d-1$, the function $\mathbf{C} \mapsto \frac{\operatorname{det} \mathbf{C}}{\operatorname{det} \mathbf{C}_{1}^{d-1}}$ is also concave (see [2], Theorem 10). The weighted version of this property is encapsulated in the following result. For a positive definite $d \times d$ matrix $\mathbf{C}$ and a WF $\mathbf{x} \mapsto \psi(\mathbf{x})$, set:

$$
\begin{aligned}
\varpi_{\psi}(\mathbf{C}): & =\frac{\alpha_{\psi}(\mathbf{C})}{2} \log \left[(2 \pi)^{d} \operatorname{det}(\mathbf{C})\right]+\frac{\log e}{2} \operatorname{tr}\left[\mathbf{C}^{-1} \boldsymbol{\Phi}_{\mathbf{C}, \psi}\right] \\
& -\frac{\alpha_{\psi_{1}^{d-1}}\left(\mathbf{C}_{1}^{d-1}\right)}{2} \log \left[(2 \pi)^{d-1} \operatorname{det}\left(\mathbf{C}_{1}^{d-1}\right)\right]-\frac{\log e}{2} \operatorname{tr}\left[\left(\mathbf{C}_{1}^{d-1}\right)^{-1} \boldsymbol{\Phi}_{\mathbf{C}_{1}^{d-1}, \mathbf{C}, \psi}\right] .
\end{aligned}
$$

Remark 6.4 When $\psi\left(\mathbf{x}_{1}^{d}\right) \equiv 1$, the expression for $\varpi_{\psi}(\mathbf{C})$ in $(\underline{6.16})$ simplifies to $\log \frac{2 \pi \text { det } \mathbf{C}}{\operatorname{det} \mathbf{C}_{1}^{d-1}}$. The aforementioned concavity property from [2], Theorem 10 (or from [5], Theorem 30), is essentially equivalent to the following subadditivity-type property:

$$
\log \frac{2 \pi \operatorname{det}(\mathbf{A}+\mathbf{B})}{\operatorname{det}\left(\mathbf{A}_{1}^{d-1}+\mathbf{B}_{1}^{d-1}\right)} \geq \log \frac{2 \pi \operatorname{det} \mathbf{A}}{\operatorname{det} \mathbf{A}_{1}^{d-1}}+\log \frac{2 \pi \operatorname{det} \mathbf{B}}{\operatorname{det} \mathbf{B}_{1}^{d-1}} .
$$

The WE-version of this property is more involved: see Eqns (6.17) - (6.19). A crucial part is played by Lemma 4.3, with $X$ represented by the random variable $Z_{d} \sim f_{A_{d d}+B_{d d}}^{\mathrm{No}}$ and $Y$ is associated with the independent Gaussian pair of vectors $\left(\mathbf{X}_{1}^{d-1}, \mathbf{Y}_{1}^{d-1}\right)$ having the joint PDF

$$
f_{\mathbf{X}_{1}^{d-1}, \mathbf{Y}_{1}^{d-1}}\left(\mathbf{x}_{1}^{d-1}, \mathbf{y}_{1}^{d-1}\right)=f_{\mathbf{A}_{1}^{d-1}}^{\mathrm{No}}\left(\mathbf{x}_{1}^{d-1}\right) f_{\mathbf{B}_{1}^{d-1}}^{\mathrm{No}}\left(\mathbf{y}_{1}^{d-1}\right) \text {. }
$$

The random element $Z$ from Theorem 4.3 is represented by $\mathbf{Z}_{1}^{d-1}$, and the map $\xi$ takes $\left(\mathbf{x}_{1}^{d-1}, \mathbf{y}_{1}^{d-1}\right) \mapsto \mathbf{x}_{1}^{d-1}+\mathbf{y}_{1}^{d-1}$.

Theorem 6.5 (Cf. [2], Theorem 10 or [5], Theorem 30.) Let A, B be two positive definite $d \times d$ matrices and $\mathbf{X} \sim f_{\mathbf{A}}^{\mathrm{No}}, \mathbf{Y} \sim f_{\mathbf{B}}^{\text {No }}$ be the corresponding independent Gaussian vectors, with $\mathbf{Z}:=\mathbf{X}+\mathbf{Y} \sim f_{\mathbf{A}+\mathbf{B}}^{\mathrm{No}}$. Consider $a W F$ $\left(z_{d}, \mathbf{x}_{1}^{d-1}, \mathbf{y}_{1}^{d-1}\right) \in \mathbb{R} \times \mathbb{R}^{d-1} \times \mathbb{R}^{d-1} \mapsto \phi\left(z_{d}, \mathbf{x}_{1}^{d-1}, \mathbf{y}_{1}^{d-1}\right)$ and assume the following inequality involving conditional normal PDFs $f_{Z_{d} \mid \mathbf{X}_{1}^{d-1}, \mathbf{Y}_{1}^{d-1}}$ and $f_{Z_{d} \mid \mathbf{Z}_{1}^{d-1}}$ :

$$
\begin{aligned}
& \int_{\mathbb{R} \times \mathbb{R}^{d-1} \times \mathbb{R}^{d-1}} \phi\left(z_{d}, \mathbf{x}_{1}^{d-1}, \mathbf{y}_{1}^{d-1}\right) f_{\mathbf{A}_{1}^{d-1}}^{\mathrm{No}}\left(\mathbf{x}_{1}^{d-1}\right) f_{\mathbf{B}_{1}^{d-1}}^{\mathrm{No}}\left(\mathbf{y}_{1}^{d-1}\right)\left[f_{Z_{d} \mid \mathbf{X}_{1}^{d-1}, \mathbf{Y}_{1}^{d-1}}\left(z_{d} \mid \mathbf{x}_{1}^{d-1}, \mathbf{y}_{1}^{d-1}\right)\right. \\
& \left.-f_{Z_{d} \mid \mathbf{Z}_{1}^{d-1}}\left(z_{d} \mid \mathbf{x}_{1}^{d-1}+\mathbf{y}_{1}^{d-1}\right)\right] \mathrm{d} z_{d} \mathrm{~d} \mathbf{x}_{1}^{d-1} \mathbf{y}_{1}^{d-1} \geq 0 .
\end{aligned}
$$

Then

$$
\varpi_{\psi}(\mathbf{A}+\mathbf{B}) \geq \varpi_{\chi}(\mathbf{A})+\varpi_{\gamma}(\mathbf{B})
$$


Here

$$
\begin{gathered}
\psi\left(\mathbf{z}_{1}^{d}\right)=\int_{\mathbb{R}^{d}} \phi\left(z_{d}-y_{d}, \mathbf{z}_{1}^{d-1}-\mathbf{y}_{1}^{d-1}\right) \frac{f_{\mathbf{A}}^{\mathrm{No}}\left(\mathbf{z}_{1}^{d}-\mathbf{y}_{1}^{d}\right) f_{\mathbf{B}}^{\mathrm{No}}\left(\mathbf{y}_{1}^{d}\right)}{f_{\mathbf{A}+\mathbf{B}}^{\mathrm{No}}\left(\mathbf{z}_{1}^{d}\right)} \mathrm{d} \mathbf{y}_{1}^{d}, \\
\chi\left(\mathbf{x}_{1}^{d}\right)=\int_{\mathbb{R}^{d}} \psi\left(\mathbf{x}_{1}^{d}+\mathbf{y}_{1}^{d}\right) f_{\mathbf{B}}^{\mathrm{No}}\left(\mathbf{y}_{1}^{d}\right) \mathrm{d} \mathbf{y}_{1}^{d}, \quad \gamma\left(\mathbf{x}_{1}^{d}\right)=\int_{\mathbb{R}^{d}} \psi\left(\mathbf{x}_{1}^{d}+\mathbf{y}_{1}^{d}\right) f_{\mathbf{A}}^{\mathrm{No}}\left(\mathbf{y}_{1}^{d}\right) \mathrm{d} \mathbf{y}_{1}^{d} .
\end{gathered}
$$

Proof. As in [2], we use basic properties of Gaussian random variables. Assume $\mathbf{X} \sim f_{\mathbf{A}}^{\text {No }}$ and $\mathbf{Y} \sim f_{\mathbf{B}}^{\text {No }}$ are independent Gaussian random vectors and set $\mathbf{Z}=\mathbf{X}+\mathbf{Y} \sim f_{\mathbf{A}+\mathbf{B}}^{\text {No }}$. By virtue of (3.6) and Theorem 4.3, we can write:

$$
h_{\psi}^{\mathrm{w}}\left(Z_{d} \mid \mathbf{Z}_{1}^{d-1}\right)=h_{\psi}^{\mathrm{w}}(\mathbf{Z})-h_{\psi_{1}^{d-1}}^{\mathrm{w}}\left(\mathbf{Z}_{1}^{d-1}\right)=\varpi(\mathbf{A}+\mathbf{B}) \geq h_{\phi}^{\mathrm{w}}\left(Z_{d} \mid \mathbf{X}_{1}^{d-1}, \mathbf{Y}_{1}^{d-1}\right) .
$$

Next, owing to independence of $\mathbf{X}$ and $\mathbf{Y}$, the conditional WE $h_{\phi}^{\mathrm{w}}\left(X_{d}+Y_{d} \mid \mathbf{X}_{1}^{d-1}, \mathbf{Y}_{1}^{d-1}\right)$ equals the sum

$$
\begin{aligned}
& \int_{\mathbb{R}^{d-1}} \chi_{1}^{d-1}\left(\mathbf{x}_{1}^{d-1}\right) f_{\mathbf{X}_{1}^{d-1}}\left(\mathbf{x}_{1}^{d-1}\right)\left\{\frac{1}{2} \log \left(\frac{2 \pi}{A_{d d}^{(-1)}}\right) \int_{\mathbb{R}} \chi_{d}(x) f_{X_{d} \mid \mathbf{X}_{1}^{d-1}}\left(x \mid \mathbf{x}_{1}^{d-1}\right) \mathrm{d} x\right. \\
& \left.+\frac{\log e}{2} A_{d d}^{(-1)} \int_{\mathbb{R}} x^{2} \chi_{d}(x) f_{X_{d} \mid \mathbf{X}_{1}^{d-1}}\left(x \mid \mathbf{x}_{1}^{d-1}\right) \mathrm{d} x\right\} \\
& +\int_{\mathbb{R}^{d-1}} \gamma_{1}^{d-1}\left(\mathbf{x}_{1}^{d-1}\right) f_{\mathbf{Y}_{1}^{d-1}}\left(\mathbf{x}_{1}^{d-1}\right)\left\{\frac{1}{2} \log \left(\frac{2 \pi}{B_{d d}^{(-1)}}\right) \int_{\mathbb{R}} \gamma_{d}(x) f_{Y_{d} \mid \mathbf{Y}_{1}^{d-1}}\left(x \mid \mathbf{x}_{1}^{d-1}\right) \mathrm{d} x\right. \\
& \left.+\frac{\log e}{2} B_{d d}^{(-1)} \int_{\mathbb{R}} x^{2} \gamma_{d}(x) f_{Y_{d} \mid \mathbf{Y}_{1}^{d-1}}\left(x \mid \mathbf{x}_{1}^{d-1}\right) \mathrm{d} x\right\} \text {. }
\end{aligned}
$$

(The fact that $X_{d}$ and $Y_{d}$ are scalar Gaussian variables is crucial here.)

The first summand equals

$$
\frac{1}{2} \log \left(\frac{2 \pi}{A_{d d}^{(-1)}}\right) \int_{\mathbb{R}^{d-1}} \chi\left(\mathbf{x}_{1}^{d}\right) f_{\mathbf{X}_{1}^{d}}\left(\mathbf{x}_{1}^{d}\right) \mathrm{d} \mathbf{x}_{1}^{d}+\frac{\log e}{2} A_{d d}^{(-1)} \int_{\mathbb{R}^{d-1}} \chi\left(\mathbf{x}_{1}^{d}\right) x_{d}^{2} f_{\mathbf{X}_{1}^{d}}\left(\mathbf{x}_{1}^{d}\right) \mathrm{d} \mathbf{x}_{1}^{d}
$$

and coincides with

$$
\begin{aligned}
& h_{\chi}^{\mathrm{w}}\left(X_{d} \mid \mathbf{X}_{1}^{d-1}\right)=\frac{\alpha_{\chi}(\mathbf{A})}{2} \log \left[(2 \pi)^{d} \operatorname{det} \mathbf{A}\right]+\frac{\log e}{2} \operatorname{tr} \mathbf{A}^{-1} \boldsymbol{\Phi}_{\mathbf{A}, \chi} \\
& -\frac{\alpha_{\chi_{1}^{d-1}}\left(\mathbf{A}_{1}^{d-1}\right)}{2} \log \left[(2 \pi)^{d-1} \operatorname{det} \mathbf{A}_{1}^{d-1}\right]-\frac{\log e}{2} \operatorname{tr}\left[\left(\mathbf{A}_{1}^{d-1}\right)^{-1} \boldsymbol{\Phi}_{\mathbf{A}_{1}^{d-1}, \chi_{1}^{d-1}}\right]=: \varpi_{\chi}(\mathbf{A}) .
\end{aligned}
$$

Similarly, the second summand coincides with

$$
\begin{aligned}
& h_{\gamma}^{\mathrm{w}}\left(Y_{d} \mid \mathbf{Y}_{1}^{d-1}\right)=\frac{\alpha_{\gamma}(\mathbf{B})}{2} \log \left[(2 \pi)^{d} \operatorname{det} \mathbf{B}\right]+\frac{\log e}{2} \operatorname{tr} \mathbf{B}^{-1} \boldsymbol{\Phi}_{\mathbf{B}, \gamma} \\
& \quad-\frac{\alpha_{\gamma_{1}^{d-1}}\left(\mathbf{B}_{1}^{d-1}\right)}{2} \log \left[(2 \pi)^{d-1} \operatorname{det} \mathbf{B}_{1}^{d-1}\right]-\frac{\log e}{2} \operatorname{tr}\left[\left(\mathbf{B}_{1}^{d-1}\right)^{-1} \mathbf{\Psi}_{\mathbf{B}_{1}^{d-1}, \gamma_{1}^{d-1}}\right]=: \varpi_{\gamma}(\mathbf{B}) .
\end{aligned}
$$

We therefore obtain the property claimed in (6.18): $\varpi_{\psi}(\mathbf{A}+\mathbf{B}) \geq \varpi_{\chi}(\mathbf{A})+\varpi_{\gamma}(\mathbf{B})$.

Finally, combining (5.23) and (6.1), we offer 
Theorem 6.6 (Cf. [5], Corollary 4) Given a $d \times d$ positive definite matrix $\mathbf{C}$, assume condition (5.20). Then

$$
\begin{aligned}
& \alpha(\mathbf{C}) \log {\left[\prod_{i=1}^{d} \frac{2 \pi(\operatorname{det} \mathbf{C})}{\operatorname{det} \mathbf{C}\left(I_{1}^{i-1} \cup I_{i+1}^{d}\right)}\right] } \\
&+\log e \sum_{i=1}^{d}\left\{\operatorname{tr}\left[\mathbf{C}^{-1} \mathbf{\Phi}\right]-\operatorname{tr}\left[\mathbf{C}\left(I_{1}^{i-1} \cup I_{i+1}^{d}\right)^{-1} \mathbf{\Phi}\left(I_{1}^{i-1} \cup I_{i+1}^{d}\right)\right]\right\} \\
& \leq \alpha(\mathbf{C}) \log \operatorname{det} \mathbf{C}-(\log e) \operatorname{tr} \mathbf{C}^{-1} \mathbf{\Phi} \leq \alpha(\mathbf{C}) \log \prod_{i} \mathbf{C}_{i i}+(\log e) \sum_{i} \mathbf{C}_{i i}^{-1} \Phi_{i i} .
\end{aligned}
$$

Acknowledgement. YS thanks the Math Department, Penn State University, for the financial support and hospitality during the academic year 2014-5. SYS thanks the CAPES PNPD-UFSCAR Foundation for the financial support in the year 2014-5. SYS thanks the Federal University of Sao Carlos, Department of Statistics, for hospitality in 2014-5. IS has been supported by FAPESP Grant - process No 11/51845-5, and expresses her gratitude to

IMS, University of São Paulo and to Math Department, University of Denver, for the warm hospitality.

\section{References}

[1] M. Belis, S. Guiasu. A Quantitative and qualitative measure of information in cybernetic systems. IEEE Trans. on Inf. Theory, 14 (1968), 593-594.

[2] T. Cover, J. Thomas. Determinant inequalities via information theory. Siam J. Matrix Anal. Appl., 9 (1988), no. 3, 384-392.

[3] T. Cover, J. Thomas. Elements of Information Theory. New York: Wiley, 2006.

[4] G. Dahlquist and A. Björck, Numerical Methods, Prentice-Hall, Englewood Cliffs, NJ, 1974, p. 161).

[5] A. Dembo, T. Cover, J.A. Thomas. Information-theoretic inequalities. IEEE Trans. Inform. Theory, 37 (6) (1991), 1501-1518.

[6] M. Kelbert. Y. Suhov. Information Theory and Coding by Example. Cambridge: CUP, 2013.

[7] K.S. Miller, On the inverse of the sum of matrices. Math. Magazine, 54, no.2, (1981), 67-72.

[8] M. Moslehian. Ky Fan inequalities. arXiv:1467v2, 2011.

[9] Y. Suhov, S. Yasaei Sekeh. Simple inequalities for weighted entropies. arXiv: 1409.4102, 2014.

[10] Y. Suhov, S. Yasaei Sekeh. An extension of the Ky Fan inequality. arXiv: 1504.01166, 2015.

[11] Y. Suhov, S. Yasaei Sekeh, M. Kelbert. Entropy-power inequality for weighted entropy. arXiv: 1502.02188v3, 2016. 